\title{
Resource Allocation for Green Cognitive Radios: Energy Efficiency Maximization
}

\author{
Zhou Yang, Wenqian Jiang $\mathbb{D}^{D}$, and Gang Li \\ Guangxi Power Grid Co., Power Grid Electric Power Research Institute, Nanning, China \\ Correspondence should be addressed to Wenqian Jiang; jwqqsky@126.com
}

Received 20 March 2018; Revised 26 April 2018; Accepted 10 May 2018; Published 5 July 2018

Academic Editor: Zheng Chu

Copyright (c) 2018 Zhou Yang et al. This is an open access article distributed under the Creative Commons Attribution License, which permits unrestricted use, distribution, and reproduction in any medium, provided the original work is properly cited.

\begin{abstract}
Green cognitive radios are promising in future wireless communications due to high energy efficiency. Energy efficiency maximization problems are formulated in delay-insensitive green cognitive radio and delay-sensitive green cognitive radio. The optimal resource allocation strategies for delay-insensitive green cognitive radio and delay-sensitive green cognitive radio are designed to maximize the energy efficiency of the secondary user. The peak interference power and the average/peak transmit power constraints are considered. Two algorithms based on the proposed resource allocation strategies are proposed to solve the formulated problems. Simulation results show that the maximum energy efficiency of the secondary user achieved under the average transmit power constraint is higher than that achieved under the peak transmit power constraint. It is shown that the design of green cognitive radio should take the tradeoff between its complexity and its achievable maximum energy efficiency into consideration.
\end{abstract}

\section{Introduction}

The unprecedented increase of mobile devices and escalating high data rate requirements have resulted in the rapid growth of energy consumption and greenhouse gas emission. It is reported in [1-3] that $2 \%$ to $10 \%$ of the global energy consumption and $2 \%$ of the greenhouse gas are generated by information and communication technologies. However, according to the Federal Communications Commission (FCC), there are $70 \%$ unused spectrum bands in the allocated spectrum bands in the USA [4-6]. Green cognitive radio (CR) is a promising and increasingly attractive technology that can improve spectrum efficiency (SE) and maximize energy efficiency (EE) simultaneously $[7,8]$. In green CR, a secondary user (SU) is allowed to access a primary user (PU) band providing that the interference caused to the PU is acceptable, and the $\mathrm{SU}$ achieves high performance with the objective of EE maximization. In this paper, a green CR under spectrum sharing is our focus.

In CR, resource allocation is of great importance and has received wide attention [9-13]. An optimal resource allocation strategy for CR not only provides the SU a reasonably high transmission rate with limited power, but also well protects the PU from harmful interference. Moreover, in green $\mathrm{CR}$, an optimal resource allocation strategy can guarantee that the $\mathrm{SU}$ achieves maximum $\mathrm{EE}$ and reduces greenhouse emission. Although the designs of the resource allocation strategies have been well studied in non-CR and CR networks, the optimal resource allocation strategies designing for non-CR and CR networks are not optimal for green CR in terms of EE maximization. Thus, it is important to design resource allocation strategies for maximizing the $\mathrm{EE}$ of the $\mathrm{SU}$ in green $\mathrm{CR}$.

1.1. Related Works and Motivation. Since the operation of a CR should protect the quality of service $(\mathrm{QoS})$ of the PU, a metric that evaluates the performance of the protection of the PU should be imposed. Basically, there are three metrics for protecting the PU from intolerance interference caused by the SU, namely, a peak interference power constraint (PIP), an average interference power (AIP) constraint, and an outage probability (OP) constraint [9-13]. A PIP constraint requires that the instantaneous interference power caused by the SU is below a prescribed interference power threshold, whereas the AIP constraint regulates the long-term interference power over all fading states to be not more than the maximum 
tolerably AIP of the PU $[9,10]$. The OP constraint requires that the transmission $\mathrm{OP}$ of the PU due to its fading and the interference from the $\mathrm{SU}$ is not greater than its acceptable target [11-13]. When the AIP constraint or the OP constraint is applied, the designs of the optimal resource allocation strategies for $\mathrm{CR}$ are required to compute a parameter with respect to the AIP constraint or the OP constraint, such as a nonnegative dual variable [9-13]. However, the computation of the parameter is complex, especially when there are several parameters (such as several nonnegative dual variables) required for computing or when the probability distribution of the fading channel gain is complex $[9,10]$. This high complexity computation consumes too much energy, which contradicts with the perspective of green CR. In contrast, it is not required to compute the parameter when the PIP constraint is used. In this paper, the PIP constraint is identified as the protection metric of the PU, which is more appropriate to green $\mathrm{CR}$ since it can facilitate the implementation of a green CR with low complexity.

The optimal resource allocation strategies have been well studied in CR with the spectrum sharing paradigm [9-18]. The designs of the optimal resource allocation strategies for CR networks combining different transmit power constraints and different interference power constraints were proposed in $[9,10]$, and those designs were extended to CR with imperfect CSI in [12]. In [11, 13], optimal resource allocation strategies were designed for $\mathrm{CR}$ with perfect CSI or CR with imperfect CSI, under an OP constraint. The ergodic capacity (EC), OC, and minimum-rate capacity based on the proposed optimal power control strategies were analyzed in [14]. In [15], the OP of a SU in spectrum sharing CR was minimized based on an optimal transmit power allocation scheme. Recently, adaptive gradient-based methods for power allocation in OFDMbased spectrum sharing CR have been proposed in [16]. Under both peak/average transmit power (PTP/ATP) and PIP/AIP constraints, optimal power control strategies that maximize the rates of $\mathrm{CR}$ with arbitrary input distributions were studied in [17]. In [18], the resource allocation problem was studied based on the proposed worst-case selective robust model.

There are some investigations of the design of the optimal resource allocation strategies for green CR [19-25]. The optimal resource allocation strategies for OFDM-based cognitive radio networks were proposed to maximize the $\mathrm{EE}$ of the SU in $[19,20]$. An EE joint relay selection and power allocation scheme was proposed in [21]. The optimal relay selection and power allocation policy is determined using a distributed approach. In [22], the optimal power control that maximizes the $\mathrm{EE}$ of green $\mathrm{CR}$ with the opportunistic spectrum access paradigm was determined. However, the optimal power allocation strategies proposed in [19-22] are not adapted to green $\mathrm{CR}$ with fading channels since the EE of green CR with fading channels should be maximized based on all fading CSI instead of instantaneous CSI. Recently, although EE maximization problems have been analyzed in fading CR channels and the optimal power allocation strategies were proposed in [23-25], the EE maximization problem has not fully taken the impact of the fading CSI between the PU's transmitter and the SU's receiver into consideration in [23]. In [24], the authors have studied secure EE maximization in green CR. Refs. [23-25] only considered EE maximization problem in delay-insensitive CR. Recently, in [26], the authors have studied the EE maximization problem in a device-to-device network. However, it did not consider the delay requirement. To our best knowledge, the EE maximization problem has not been analyzed in delaysensitive CR.

Recently, the EE maximization problems were studied in delay-insensitive $\mathrm{CR}$, delay-sensitive $\mathrm{CR}$, and simultaneously delay-sensitive and delay-insensitive CR in [27]. In [27], the AIP constraint was regarded as the metric of the protection of the PU. Similar to the optimal power allocation strategies for conventional CR proposed in [9-18], which use the AIP constraint as the metric of the protection of the PU, the proposed optimal power allocation strategies for green CR also need to compute a complex nonnegative dual variable related to the AIP constraint. The computation of the nonnegative dual variable is extensively complex when there are several nonnegative dual variables required to simultaneously compute or when the probability distributions of the fading channels involved are complex. This complex computation limits its application when green CR requires fast and efficient determination of the optimal power allocation strategies. In this paper, different from the works in [25, 27], a PIP constraint is identified as the metric protecting the PU from harmful interference caused by the SU. EE maximization problems subject to the PIP constraint are analyzed in delayinsensitive green CR and delay-sensitive green CR. Optimal power allocation strategies that maximize the $\mathrm{EE}$ of the $\mathrm{SU}$ under the PIP constraint are found. The proposed optimal power allocation strategies have advantages in low complexity and fast computation.

1.2. Contributions and Organization. Different from the works in $[25,27]$, EE maximization problems subject to the PIP constraint are proposed in delay-insensitive green CR and delay-sensitive green $\mathrm{CR}$, where the SU coexists with the PU and all the channels involved are fading. The EE maximization problem is first analyzed in delay-insensitive green CR. Then, the EE maximization problem is extended to delay-sensitive green CR. The main contributions of this work are summarized as follows.

(1) The optimal resource allocation strategies for delayinsensitive green $\mathrm{CR}$ and delay-sensitive green $\mathrm{CR}$ that maximize the EE of the SU are found. Different from the work in $[25,27]$, the PIP constraint, along with the ATP/PTP constraint, is considered. It is shown that the optimal power allocation strategies for delay-insensitive green CR have similar form to the well-known "water-filling" power allocation strategies and that the optimal power allocation strategies for delay-sensitive green $\mathrm{CR}$ have similar form to the well-known truncated channel inversion power allocation strategies.

(2) Two algorithms based on the proposed optimal resource allocation strategies are presented. One is proposed to solve the EE maximization problem 


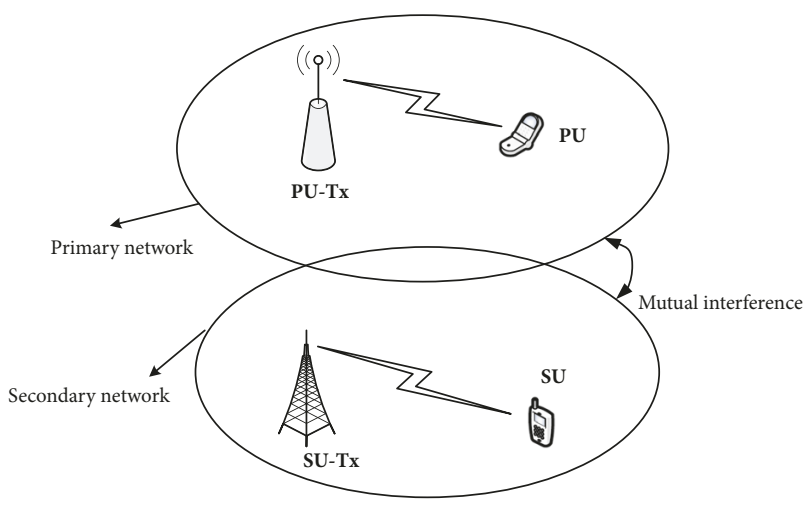

FIGURE 1: The system model.

when the ATP constraint and the PIP constraint are applied. The other one is given for solving the EE maximization when the PTP constraint and the PIP constraint are imposed. It is shown that the complexity of the proposed algorithm for the EE maximization problem subject to the ATP constraint is higher than that of the proposed algorithm for the EE maximization problem subject to the PTP constraint.

(3) Simulation results show that the maximum EE of the SU achieved under the ATP constraint is larger than that achieved under the PTP constraint. The design of a green CR system should take the tradeoff between the achievable maximum EE and the implementation complexity into consideration.

The rest of this paper is organized as follows. Section 2 presents the system model. The EE maximization problem subject to constraints on the PIP and the ATP is presented in Section 3. Section 4 analyzes the EE maximization problem subject to the PIP and the PTP constraint. Section 5 presents simulation results. The paper concludes with Section 6 .

\section{System Model}

As shown in Figure 1, a cognitive radio consisting of one $\mathrm{PU}$ and one SU is considered. The SU coexists with the $\mathrm{PU}$ under the spectrum sharing paradigm. There is one PU transmitter (PU-Tx) and one PU in the primary network while the secondary network has one SU transmitter (SU$\mathrm{Tx}$ ) and one SU. It is assumed that all the terminals have one antenna. The channel between the PU-Tx and the PU, the channel between the PU-Tx and the SU, the channel between the SU-Tx and the SU, and the channel between the SU-Tx and the PU are assumed to be block fading. The corresponding channel power gains at fading state $v$ are denoted by $g_{s s}(\nu), g_{s p}(\nu), h_{p p}(\nu)$, and $h_{p s}(\nu)$, respectively. The fading index for all related channels is denoted by $v$. All the channel power gains are assumed to be independent identically distributed (i.i.d.), ergodic, and stationary and have continuous probability density functions. It is assumed that perfect channel state information (CSI) related to $g_{s s}(\nu)$, $g_{s p}(\nu)$, and $h_{p p}(\nu)$ is available to the SU-Tx. The additive white Gaussian noise (AWGN) at the SU-Rx is circularly symmetric complex AWGN with mean zero and variance $\sigma_{w}^{2}$. It is assumed that the PU does not have knowledge of the CSI of the PU link, and thus it transmits with a constant power, denoted by $P_{p}$.

\section{EE Maximization under Average Transmit Power Constraint}

In this section, EE maximization problems are formulated in delay-insensitive green CR and delay-sensitive green CR. Different from the works in $[25,27]$, the PIP constraint is considered. The optimal resource allocation strategies that maximize the EE of the SU, subject to the PIP constraint and the ATP constraint, are determined. An algorithm based on the proposed optimal power allocation strategy is presented to solve EE maximization problems.

3.1. EE Maximization in Delay-Insensitive Green CR. In CR, from the perspective of the PU, the interference caused by the SU should not be beyond the tolerable interference threshold of the PU. The PIP constraint is chosen as the protection metric of the PU since CR with PIP constraint has low complexity and facilitated implementation. In addition, the long-term power budget of the SU should be considered and can be evaluated by the ATP. From the perspective of the SU, the ATP should be below a threshold. Thus, the constraints on the PIP and ATP can be given as

$$
\begin{gathered}
g_{s p}(\nu) P_{s}(\nu) \leq P_{I n} \\
\mathbb{E}\left\{P_{s}(\nu)\right\} \leq \overline{P_{t h}} \\
P_{s}(\nu) \geq 0
\end{gathered}
$$

where $P_{s}(v)$ denotes the transmit power of the SU. $P_{I n}$ and $\overline{P_{t h}}$ are the tolerable maximum PIP of the PU and the maximum ATP of the SU, respectively. $\mathbb{E}(\cdot)$ is the expectation operator.

In delay-insensitive $\mathrm{CR}$, the EC is appropriately used to evaluate the performance of the SU [10]. The EC determines the maximum achievable long-term rates of the $S U$, which is averaged over all fading states. The EE maximization problem for delay-insensitive green CR under the PIP constraint and the ATP constraint can be formulated as problem $\mathbf{P}_{\mathbf{1}}$, given as

$$
\begin{aligned}
\mathbf{P}_{\mathbf{1}}: \max _{P_{s}(v)} & \eta_{E E}\left(P_{s}(\nu)\right)=\frac{\mathbb{E}\left\{\log _{2}\left(1+g_{s s}(\nu) P_{s}(\nu) /\left(h_{p s}(v) P_{p}+\sigma_{w}^{2}\right)\right)\right\}}{\mathbb{E}\left\{\zeta P_{s}(v)+P_{C}\right\}} \\
\text { s.t. } & (1) \text { and (2a), (2b) are satisfied }
\end{aligned}
$$


where $\eta_{E E}\left(P_{s}(\nu)\right)$ is the energy efficient function of $P_{s}(\nu) . \zeta$ and $P_{C}$ represent the amplifier coefficient $(1 / \zeta$ is also known as the power efficiency for the power amplifier) and the constant circuit power consumption of SU-Tx, respectively. Let $S_{1}$ denote the set $S_{1}=\left\{P_{s}(\nu) \mid P_{s}(\nu) \in(1), P_{s}(\nu) \in(2 \mathrm{a})\right.$, (2b)\}. Note that $S_{1}$ is a convex set. According to the fractional programming theory given in $[28,29]$, Theorem 1 can be stated as follows.

Theorem 1. Problem $\mathbf{P}_{1}$ is a nonlinear strictly quasiconcave and strictly pseudoconcave fractional programming problem since the numerator of $\eta_{E E}\left(P_{s}(\nu)\right)$ is strictly concave and differentiable, the denominator of $\eta_{E E}\left(P_{s}(\nu)\right)$ is affine and differentiable, and the denominator $\mathbb{E}\left\{\zeta P_{s}(\nu)+P_{C}\right\}>0$. Any local maximum of problem $\mathbf{P}_{\mathbf{1}}$ is a global maximum of problem $\mathbf{P}_{\mathbf{1}}$, and $\mathbf{P}_{\mathbf{1}}$ has at most one maximum since $\mathbf{P}_{\mathbf{1}}$ is strictly quasiconcave.

Proof. See Appendix A.

Based on Theorem 1 and Dinkelbach's method [30], problem $\mathbf{P}_{\mathbf{1}}$ is equivalent to the parameter optimization problem, denoted by $\mathbf{P}_{\mathbf{2}}$, given as

$$
\begin{array}{rl}
\mathbf{P}_{2}: \max _{P_{s}(\nu) \in S_{1}} & f(\eta) \\
= & \mathbb{E}\left\{\log _{2}\left(1+\frac{g_{s s}(\nu) P_{s}(\nu)}{h_{p s}(\nu) P_{p}+\sigma_{w}^{2}}\right)\right\} \\
& -\eta \mathbb{E}\left\{\zeta P_{s}(\nu)+P_{C}\right\}
\end{array}
$$

where $\eta$ is a nonnegative parameter. The following theorem can be obtained to solve problem $\mathbf{P}_{\mathbf{1}}$ and $\mathbf{P}_{\mathbf{2}}$.

Theorem 2. The global optimization solution of problem $\mathbf{P}_{\mathbf{1}}$ is achieved if and only if

$$
\begin{aligned}
\max _{P_{s}(\nu) \in S_{1}} f\left(\eta_{o p t}\right) \\
=\mathbb{E}\left\{\log _{2}\left(1+\frac{g_{s s}(\nu) P_{s}^{o p t}(\nu)}{h_{p s}(\nu) P_{p}+\sigma_{w}^{2}}\right)\right\} \\
-\eta_{o p t} \mathbb{E}\left\{\zeta P_{s}^{o p t}(\nu)+P_{C}\right\}=0 .
\end{aligned}
$$

$\eta_{o p t}$ is the optimal solution of $\mathbf{P}_{\mathbf{2}}$ and also is the maximum EE of $\mathbf{P}_{\mathbf{1}} . P_{s}^{\text {opt }}(\nu)$ is the optimal transmit power of problem $\mathbf{P}_{\mathbf{1}}$ and is the corresponding solution of $\eta_{o p t}$.

Proof. See Appendix B.

Thus, on the one hand, problem $\mathbf{P}_{\mathbf{1}}$ can be solved by solving problem $\mathbf{P}_{2}$. On the other hand, for a given $\eta_{o p t}$, the optimal transmit power can be obtained by solving (5).

For a given $\eta$, problem $\mathbf{P}_{2}$ can be solved by using the Lagrange duality method [31]. The Lagrangian with respective to the transmit power $P_{s}(\nu)$ of $\mathbf{P}_{2}$ is given as

$$
\begin{aligned}
& L\left(P_{s}(\nu), \tau\right)= \mathbb{E} \\
&\left\{\log _{2}\left(1+\frac{g_{s s}(\nu) P_{s}(\nu)}{h_{p s}(\nu) P_{p}+\sigma_{w}^{2}}\right)\right\} \\
&-\eta \mathbb{E}\left\{\zeta P_{s}(\nu)+P_{C}\right\} \\
&-\tau\left\{\mathbb{E}\left\{P_{s}(\nu)\right\}-\overline{P_{t h}}\right\}
\end{aligned}
$$

where $\tau$ is the nonnegative dual variable with respect to (2a). Let $S_{2}$ denote the set $S_{2}=\left\{P_{s}(\nu) \mid 0 \leq P_{s}(\nu) \leq P_{I n} / g_{s p}(\nu)\right\}$. Then, the Lagrange dual function of $\mathbf{P}_{2}$ can be presented as

$$
g(\tau)=\max _{P_{s}(\nu) \in S_{2}, \forall \nu} L\left(P_{s}(\nu), \tau\right) .
$$

Similar to $[9,11]$, the problem given by (7) can be decoupled into parallel subproblems by using the Lagrange dualdecomposition method [31]. Thus, the corresponding subproblem for a fading state can be given as

$$
\max _{P_{s} \in S_{2}} y\left(P_{s}\right)=\log _{2}\left(1+\frac{g_{s s} P_{s}}{h_{p s} P_{p}+\sigma_{w}^{2}}\right)-\eta \zeta P_{s}-\tau P_{s} .
$$

In order to solve $\mathbf{P}_{\mathbf{2}},(8)$ is required to iteratively solve for all fading states with respect to fixed $\tau$ and updating $\tau$. $\tau$ can be updated by using the subgradient method [31], given as

$$
\tau^{k+1}=\left[\tau^{k}-t_{1}\left(\overline{P_{t h}}-\mathbb{E}\left\{P_{s, k}^{o p t}\right\}\right)\right]^{+}
$$

where $[a]^{+}=\max (a, 0)$ and $k$ is the iteration index. $P_{s, k}^{o p t}$ denotes the optimal power level of the $k$ th iteration of the subgradient method, and the parameter $t_{1}$ denotes the iteration step size. Since $y\left(P_{s}\right)$ is a concave function related to $P_{s}$, the optimal power allocation strategy of $\mathbf{P}_{2}$, denoted by $P_{s}^{o p t}$, can be found as given in Theorem 3. Let $\min (a, b)$ denote the minimum between $a$ and $b$.

Theorem 3. The optimal resource allocation strategy of $\mathbf{P}_{\mathbf{2}}$ is given by

$$
\begin{aligned}
\widehat{P}_{s} & =\left[\frac{1}{(\eta \zeta+\tau) \ln 2}-\frac{\left(h_{p s} P_{p}+\sigma_{w}^{2}\right)}{g_{s s}}\right]^{+} \\
P_{s}^{o p t} & =\min \left(\widehat{P}_{s}, \frac{P_{I n}}{g_{s p}}\right)
\end{aligned}
$$

Remark 4. In delay-insensitive green $\mathrm{CR}$, the optimal power allocation strategy for EE maximization should take the achievable EE of the SU and the power amplifier coefficient of the SU-Tx into consideration, which is different from the optimal power allocation strategy for EC maximization in the conventional delay-insensitive CR proposed in $[10$, $12,14]$. When $\eta=0$, problem $\mathbf{P}_{2}$ degenerates into the conventional EC maximization problem. This can be seen from (4). In this case, the optimal power allocation strategy for EE maximization subject to the PIP constraint and the ATP constraint, given by (10a) and (10b), has a similar form to that given in $[10$, eq. (13)]. The difference lies in the fact that the influence of the fading of the channel between the PU-Tx and the SU is fully considered, whereas that influence is only partially considered in [10]. 


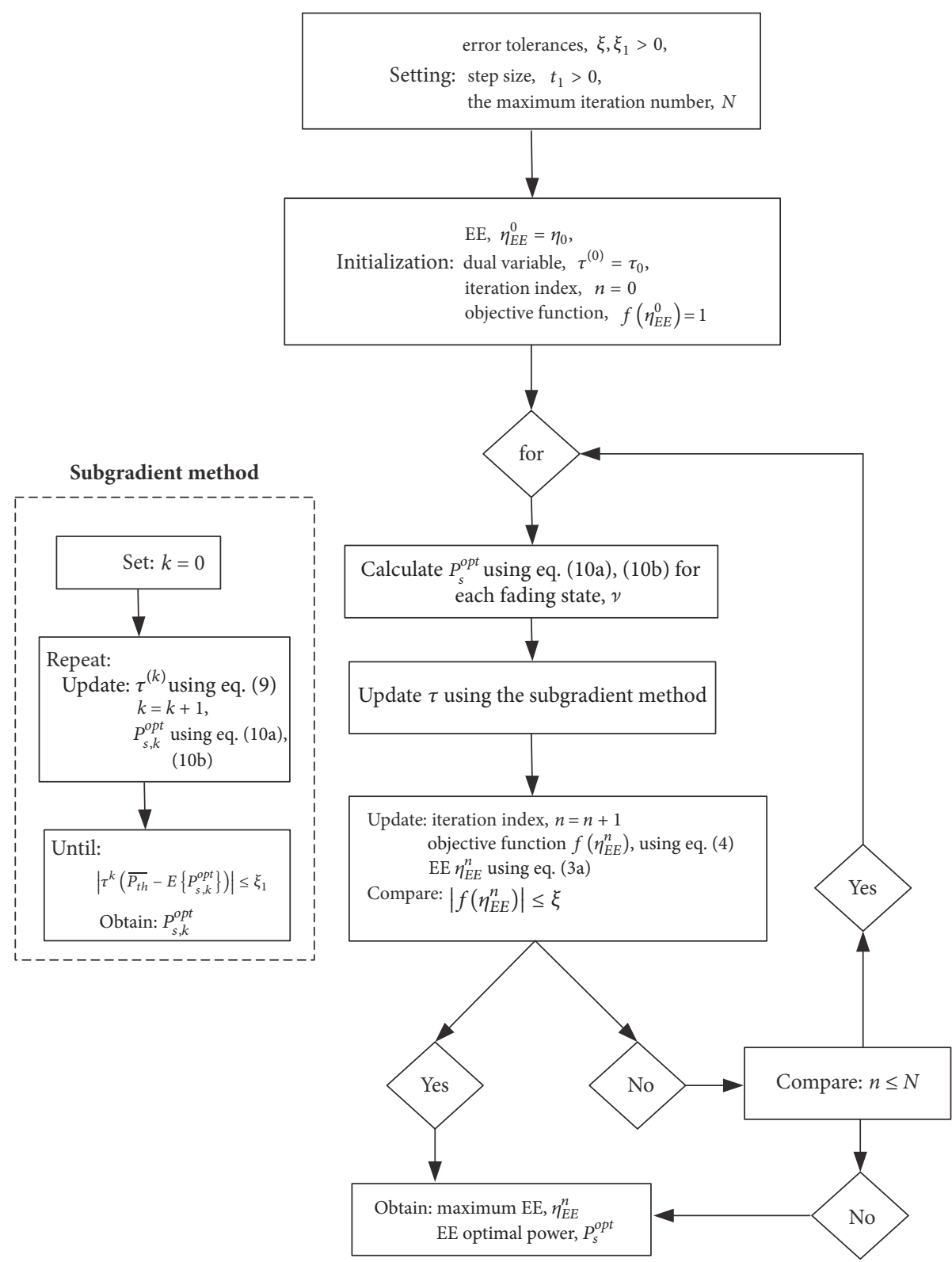

FIGURE 2: Flowchart of Algorithm 1 for EE maximization subject to the ATP constraint and the PIP constraint.

For a given $\eta, \mathbf{P}_{2}$ can be efficiently solved by using the proposed optimal power allocation strategy given by (10a) and (10b). In order to solve problem $\mathbf{P}_{\mathbf{1}}$ and obtain the maximum EE of the SU, Dinkelbach's method is applied. An algorithm based on the subgradient method and Dinkelbach's method is proposed to solve problem $\mathbf{P}_{\mathbf{1}}$, denoted by Algorithm 1 . The principle of this algorithm is as follows. The optimal transmit power is required to be iteratively updated by using Theorem 3. The subgradient method is used to compute the nonnegative dual variable until the optimal power level can satisfy the ATP constraint, namely, $\left|\tau^{k}\left(\overline{P_{t h}}-E\left\{P_{s, k}^{o p t}\right\}\right)\right| \leq \xi_{1} . k$ is the iterative index of the subgradient method and $\xi_{1}$ is the tolerable error for the ATP constraint. When the proposed optimal power allocation strategy can support $f\left(\eta_{E E}^{n}\right)=0$, the maximum EE and corresponding optimal power level of the SU are obtained. Otherwise, an $\xi$-optimal solution with an error tolerance $\xi$ is adopted. In this case, the maximum EE and corresponding optimal power level are obtained when $\left|f\left(\eta_{E E}^{n}\right)\right| \leq \xi$. Note that if equations $f\left(\eta_{E E}^{n}\right)=0$ and $\left|f\left(\eta_{E E}^{n}\right)\right| \leq$ $\xi$ can not be satisfied, Algorithm 1 stops when the iteration number becomes $N$. The flowchart of Algorithm 1 for EE maximization subject to the PIP constraint and the ATP constraint is shown in Figure 2.

3.2. EE Maximization in Delay-Sensitive Green CR. In this subsection, the EE maximization problem is analyzed in delay-sensitive green CR, subject to constraints on the PIP and the ATP. In delay-sensitive green $\mathrm{CR}$, the SU is sensitive 
to the delay, such as voice and video applications. In delaysensitive CR, the OC that evaluates the achievable constant rate for all fading states is a more appropriate metric. Thus, the EE definition in this green $\mathrm{CR}$ should be related to the OC of the SU.
According to the work in [9-13], the traditional OP minimization problem subject to the PIP constraint and the ATP constraint can be formulated as the problem, $\mathbf{P}_{\mathbf{3}}$, given as

$$
\begin{gathered}
\mathbf{P}_{3}: \min _{p_{s}(\nu) \geq 0, \forall v} \operatorname{Pr}\left\{\log _{2}\left(1+\frac{g_{s s}(\nu) P_{s}(\nu)}{h_{p s}(\nu) P_{p}+\sigma_{w}^{2}}\right)<r_{s}\right\} \\
\text { s.t. } \\
\text { (1) and (2a), (2b) are satisfied }
\end{gathered}
$$

where $r_{s}$ is the prescribed OC of the SU. It is straightforward to see that the problem $\mathbf{P}_{\mathbf{3}}$ is equivalent to the following problem, $\mathbf{P}_{4}$, given as

$$
\begin{array}{cc}
\mathbf{P}_{4}: \max _{p_{s}(v) \geq 0, \forall v} & 1-\operatorname{Pr}\left\{\log _{2}\left(1+\frac{g_{s s}(\nu) P_{s}(\nu)}{h_{p s}(\nu) P_{p}+\sigma_{w}^{2}}\right)<r_{s}\right\} \\
\text { s.t. } & \text { (1) and (2a), (2b) are satisfied. }
\end{array}
$$

According to [27], the EE in delay-sensitive green CR is given as

$$
\begin{aligned}
& \eta_{E E}\left(P_{s}(\nu)\right) \\
& =\frac{r_{s}\left\{1-\operatorname{Pr}\left\{\log _{2}\left(1+g_{s s}(\nu) P_{s}(\nu) /\left(h_{p s}(\nu) P_{p}+\sigma_{w}^{2}\right)\right)<r_{s}\right\}\right\}}{\mathbb{E}\left\{\zeta P_{s}(\nu)+P_{C}\right\}}
\end{aligned}
$$

where $\eta_{E E}\left(P_{s}(\nu)\right)$ denotes the EE function with respect to $P_{s}(\nu)$ in delay-sensitive green CR. In order to formulate the EE maximization problem, the OP given by (11a) is represented as

$$
\begin{aligned}
& \operatorname{Pr}\left\{\log _{2}\left(1+\frac{g_{s s}(\nu) P_{s}(\nu)}{h_{p s}(\nu) P_{p}+\sigma_{w}^{2}}\right)<r_{s}\right\}=E\left\{\chi_{s}(v)\right\} \\
& \chi_{s}(v)= \begin{cases}1, & \log _{2}\left(1+\frac{g_{s s}(\nu) P_{s}(\nu)}{h_{p s}(\nu) P_{p}+\sigma_{w}^{2}}\right)<r_{s} \\
0, & \text { otherwise }\end{cases}
\end{aligned}
$$

where $\chi_{s}(v)$ is an indicator function for the outage event of the SU at fading state $\nu$. Thus, the EE maximization problem subject to constraints on the PIP and the ATP can be formulated as problem, $\mathbf{P}_{5}$, given as

$$
\begin{aligned}
\mathbf{P}_{5}: \max _{P_{s}(\nu)} & \eta_{E E}\left(P_{s}(\nu)\right)=\frac{r_{s} \mathbb{E}\left\{1-\chi_{s}(v)\right\}}{\mathbb{E}\left\{\zeta P_{s}(\nu)+P_{C}\right\}} \\
\text { s.t. } & (1) \text { and }(2 \mathrm{a}),(2 \mathrm{~b}) \text { are satisfied. }
\end{aligned}
$$

Since $\chi_{s}(v)$ is not a concave function, problem $\mathbf{P}_{5}$ is a general nonlinear fractional programming problem instead of a nonlinear concave fractional programming problem.
Although problem $\mathbf{P}_{\mathbf{5}}$ is not a nonlinear concave fractional programming problem, Dinkelbach's method can nonetheless be applied to solve problem $\mathbf{P}_{\mathbf{5}}$ [30]. The reason is that the numerator and the denominator of $\eta_{E E}\left(P_{s}(\nu)\right)$ satisfy the condition of Dinkelbach's method; the numerator and the denominator of $\eta_{E E}\left(P_{s}(\nu)\right)$ are continuous and satisfy $\mathbb{E}\left\{\zeta P_{s}(\nu)+P_{C}\right\}>0$ and $\mathbb{E}\left\{1-\chi_{s}(\nu)\right\} \geq 0$ for all $P_{s}(\nu) \in S_{1}$. Thus, based on Dinkelbach's method, problem $\mathbf{P}_{5}$ can be equivalent to the parameter optimization problem, $\mathbf{P}_{\mathbf{6}}$, given as

$$
\begin{array}{rl}
\mathbf{P}_{\mathbf{6}}: \max _{P_{s}(v) \in S_{1}} & f(\eta) \\
& =r_{s} \mathbb{E}\left\{1-\chi_{s}(v)\right\}-\eta \mathbb{E}\left\{\zeta P_{s}(\nu)+P_{C}\right\}
\end{array}
$$

where $\eta$ is a nonnegative parameter. Using a similar method to that used for problem $\mathbf{P}_{2}$, problem $\mathbf{P}_{\mathbf{6}}$ can be solved by solving the following problem, given as

$$
\max _{P_{s} \in S_{2}} y\left(P_{s}\right)=-r_{s} \chi_{s}\left(P_{s}\right)-\eta \zeta P_{s}-\tau P_{s}
$$

where $\tau$ is the nonnegative dual variable with respect to the ATP constraint given by $(2 \mathrm{a})$ and $\chi_{s}\left(P_{s}\right)$ is an explicit function of $P_{s}$. Note that the fading state indicator, $v$, is dropped. Similar to problem $\mathbf{P}_{\mathbf{2}}$, problem $\mathbf{P}_{\mathbf{6}}$ can be solved by iteratively solving (17) for fixed $\tau$, and then updating $\tau$ by using the subgradient method given in $(9) . \chi_{s}\left(P_{s}\right)$ is in general a step function with respect to $P_{s}$. Let $y$ denote the turning point of $\chi_{s}\left(P_{s}\right)$, given as

$$
y=\frac{\left(2^{r_{s}}-1\right)\left(h_{p s} P_{p}+\sigma_{w}^{2}\right)}{g_{s s}}
$$


where $y \geq 0$. It is seen that $y$ is the minimum power required for the SU to guarantee the OC of the SU, $r_{s}$. Note that $\chi_{s}\left(P_{s}\right)=$ 1 for $P_{s}<y$ and $\chi_{s}\left(P_{s}\right)=0$ for $P_{s} \geq y$. Let $P_{s}^{o p t}$ denote the optimal power of $\mathbf{P}_{\mathbf{6}}$. The following results can be obtained by solving (17).

Case $1\left(y>P_{I n} / g_{s p}\right)$. In this case, when the SU transmits with the minimum power required to maintain the $\mathrm{OC} r_{s}$ of the $\mathrm{SU}$, the interference power caused for the PU is larger than the PIP constraint. The SU is always in outage if the SU transmits with the feasible power in $S_{1}$. Thus, $P_{s}^{\text {opt }}=0$.

Case $2\left(y \leq P_{\text {In }} / g_{s p}\right)$. In this case, the maximum of $y\left(P_{s}\right)$ may be $-r_{s}$ when $P_{s}=0$ or may be $-(\eta \zeta+\tau) y$ when $P_{s}=y$, which is the maximum depending on their relationship. When $y>$ $r_{s} /(\eta \zeta+\tau)$, the minimum power required to maintain the OC $r_{s}$ of the SU is very large, and the SU stops transmitting in order to save power. Thus, $P_{s}^{o p t}=0$. Otherwise, the SU transmits with the optimal power, $P_{s}^{o p t}=y$.

Theorem 5 can now be formally stated based on the results obtained above.

Theorem 5. The optimal resource allocation strategy of $\mathbf{P}_{\mathbf{6}}$ is given by

$$
P_{s}^{o p t}= \begin{cases}0, & y>\frac{P_{\text {In }}}{g_{s p}} \\ 0, & \frac{r_{s}}{\eta \zeta+\tau}<y \leq \frac{P_{I n}}{g_{s p}} \\ y, & y \leq \frac{P_{I n}}{g_{s p}}, y \leq \frac{r_{s}}{\eta \zeta+\tau} .\end{cases}
$$

Remark 6. It is seen from (16) that EE maximization problem in delay-sensitive $\mathrm{CR}$ is equivalent to the conventional $\mathrm{OP}$ minimization problem proposed in [10] when $\eta=0$. Thus, it is seen that the optimal power allocation strategy given by (19) is similar to the form given in [10, eq. (28)] when $\eta=0$. When $\eta=0$, the difference of the optimal power allocation strategy for EE maximization given by (19) from the form given in $[10$, eq. (28)] lies in the fact that the effect of the PU on the SU is fully considered in this paper while that effect is only partially considered in [10]. It is also seen that the optimal power allocation strategy for EE maximization given by (19) is influenced by the EE of the SU and the amplifier coefficient of the SU-Tx. Furthermore, when the PIP is sufficiently loose and the equation $y \leq r_{s} /(\eta \zeta+\tau)$ always holds, the optimal power level is equal to the minimum transmitted power level required to guarantee the $\mathrm{OC}$ of the $\mathrm{SU}$, and the maximum $\mathrm{EE}$ of the SU is achieved at the minimum transmitted power level required to guarantee the $\mathrm{OC}$. Intuitively, in delay-sensitive green $\mathrm{CR}$, it is reasonable that the SU does not need to increase transmitted power when the OC can be guaranteed and thus the maximum EE is achieved when the SU transmits with the minimum power level required to guarantee the OC. Finally, when the ATP constraint is sufficiently loose compared with the PIP constraint and $y \leq r_{s} /(\eta \zeta+\tau)$ always holds, it is seen that the optimal power allocation strategy is only dependent on the PIP constraint and is equivalent to the optimal power allocation strategy for OP minimization given in $[10$, eq. (28)].

It is seen that problem $\mathbf{P}_{\mathbf{6}}$ can be efficiently solved by using the proposed optimal power allocation strategy given in Theorem 5 for a given $\eta$. In order to solve problem $\mathbf{P}_{5}$ and obtain the maximum EE of the SU and the corresponding optimal power allocation strategy, Algorithm 1 can be applied and it only requires several modifications. In this case, the optimal power, $P_{s}^{o p t}$, is calculated by using Theorem 5 . The objective function, $f\left(\eta_{E E}^{n}\right)$, and EE, $\eta_{E E}^{n}$, are calculated by using (16) and (13), respectively. For brevity, the details are not given here.

\section{EE Maximization under Peak Transmit Power Constraint}

In this section, EE maximization problems subject to constraints on the PIP and the PTP are studied in delayinsensitive green CR and delay-sensitive green CR. The PTP constraint is related to the nonlinearity of power amplifiers. Another algorithm based on the derived optimal power allocation strategies is proposed to solve EE maximization problems under the PIP constraint and the PTP constraint.

4.1. EE Maximization in Delay-Insensitive Green CR. In this subsection, the peak transmit power constraint is considered, given as

$$
\begin{aligned}
& P_{s}(\nu) \leq P_{t h} \\
& P_{s}(\nu) \geq 0
\end{aligned}
$$

where $P_{t h}$ is the maximum instantaneous transmit power of the SU. In delay-insensitive green $\mathrm{CR}$, the EE maximization problem subject to the PIP constraint and the PTP constraint, denoted by $\mathbf{P}_{7}$, is given as

$$
\begin{aligned}
\mathbf{P}_{7}: \max _{P_{s}(\nu)} & \eta_{E E}\left(P_{s}(\nu)\right)=\frac{\mathbb{E}\left\{\log _{2}\left(1+g_{s s}(\nu) P_{s}(\nu) /\left(h_{p s}(\nu) P_{p}+\sigma_{w}^{2}\right)\right)\right\}}{\mathbb{E}\left\{\zeta P_{s}(\nu)+P_{C}\right\}} \\
\text { s.t. } & \text { (1) and (20a), (20b) are satisfied. }
\end{aligned}
$$

Let $S_{3}$ be $S_{3}=\left\{P_{s}(\nu) \mid P_{s}(\nu) \in(1), P_{s}(\nu) \in(20 \mathrm{a}),(20 \mathrm{~b})\right\}$. It is straightforward to show that $S_{3}$ is a convex set. And thus, problem $\mathbf{P}_{7}$ is also a nonlinear strictly quasiconcave and strictly pseudoconcave fractional programming problem. 
Based on Dinkelbach's method, problem $\mathbf{P}_{7}$ is equivalent to the parameter optimization problem, denoted by $\mathbf{P}_{\mathbf{8}}$, given as

$$
\begin{aligned}
\mathbf{P}_{\mathbf{8}}: \max _{P_{s}(\nu) \in S_{3}} f(\eta) & \\
= & \mathbb{E}\left\{\log _{2}\left(1+\frac{g_{s s}(\nu) P_{s}(\nu)}{h_{p s}(\nu) P_{p}+\sigma_{w}^{2}}\right)\right\} \\
& -\eta \mathbb{E}\left\{\zeta P_{s}(\nu)+P_{C}\right\}
\end{aligned}
$$

where $\eta$ is a nonnegative parameter. Using a similar method as used in Section 3, problem $\mathbf{P}_{\mathbf{8}}$ can be solved by solving the following problem, given as

$$
\max _{P_{s} \in S_{3}} y\left(P_{s}\right)=\log _{2}\left(1+\frac{g_{s s} P_{s}}{h_{p s} P_{p}+\sigma_{w}^{2}}\right)-\eta \zeta P_{s} .
$$

For a given $\eta$, problem $\mathbf{P}_{\mathbf{8}}$ can be solved by solving (23) for all fading states. Let $P_{s}^{o p t}$ denote the optimal power of $\mathbf{P}_{\mathbf{8}}$. Since $y\left(P_{s}\right)$ is concave with respect to $P_{s}$, the optimal power allocation strategy for a given $\eta, P_{s}^{o p t}$, can be obtained by solving (23) and is formally stated in Theorem 7 . Let $\min (a, b, c)$ denote the minimum of $a, b$, and $c$.

Theorem 7. The optimal resource allocation strategy of $\mathbf{P}_{\mathbf{8}}$ is given by

$$
\begin{aligned}
\widehat{P}_{s} & =\left[\frac{1}{\eta \zeta \ln 2}-\frac{\left(h_{p s} P_{p}+\sigma_{w}^{2}\right)}{g_{s s}}\right]^{+} \\
P_{s}^{o p t} & =\min \left(\widehat{P}_{s}, \frac{P_{I n}}{g_{s p}}, P_{t h}\right)
\end{aligned}
$$

Remark 8. When $\eta=0$, the optimal power allocation strategy for delay-insensitive green CR subject to the PIP constraint and the PTP constraint, given in Theorem 7, is equivalent to the optimal power allocation strategy for EC maximization in conventional CR given in [10, eq. (11)]. In this case, the EE maximization problem is equivalent to the EC maximization problem in conventional CR. This also can be seen from (22). From (24a) and (24b), it is seen that the optimal power allocation strategy for maximizing the achievable EE of the $\mathrm{SU}$ is required to take the $\mathrm{EE}$ of the $\mathrm{SU}$ and the amplifier coefficient into consideration. Moreover, when $\widehat{P}_{s}$ is larger than the maximum of $P_{t h}$ and $P_{I n} / g_{s p}$, it is seen from (24a) and $(24 \mathrm{~b})$ that the optimal power strategy given in Theorem 7 is also equivalent to the optimal power allocation strategy for EC maximization in conventional CR given in [10, eq. (11)].

For a given $\eta$, it is seen that problem $\mathbf{P}_{\mathbf{8}}$ can be efficiently solved by using the proposed optimal power allocation strategy given in Theorem 7 . In order to solve problem $\mathbf{P}_{\mathbf{7}}$ and obtain the maximum EE of the $\mathrm{SU}$, similar to problem $\mathbf{P}_{\mathbf{1}}$ and problem $\mathbf{P}_{5}$, Dinkelbach's method can be applied. An iterative optimal power algorithm based on Dinkelbach's method is proposed to solve problem $\mathbf{P}_{7}$, denoted by Algorithm 2. The principle of this algorithm is as follows. The optimal power level is iteratively updated by using Theorem 7 . The maximum

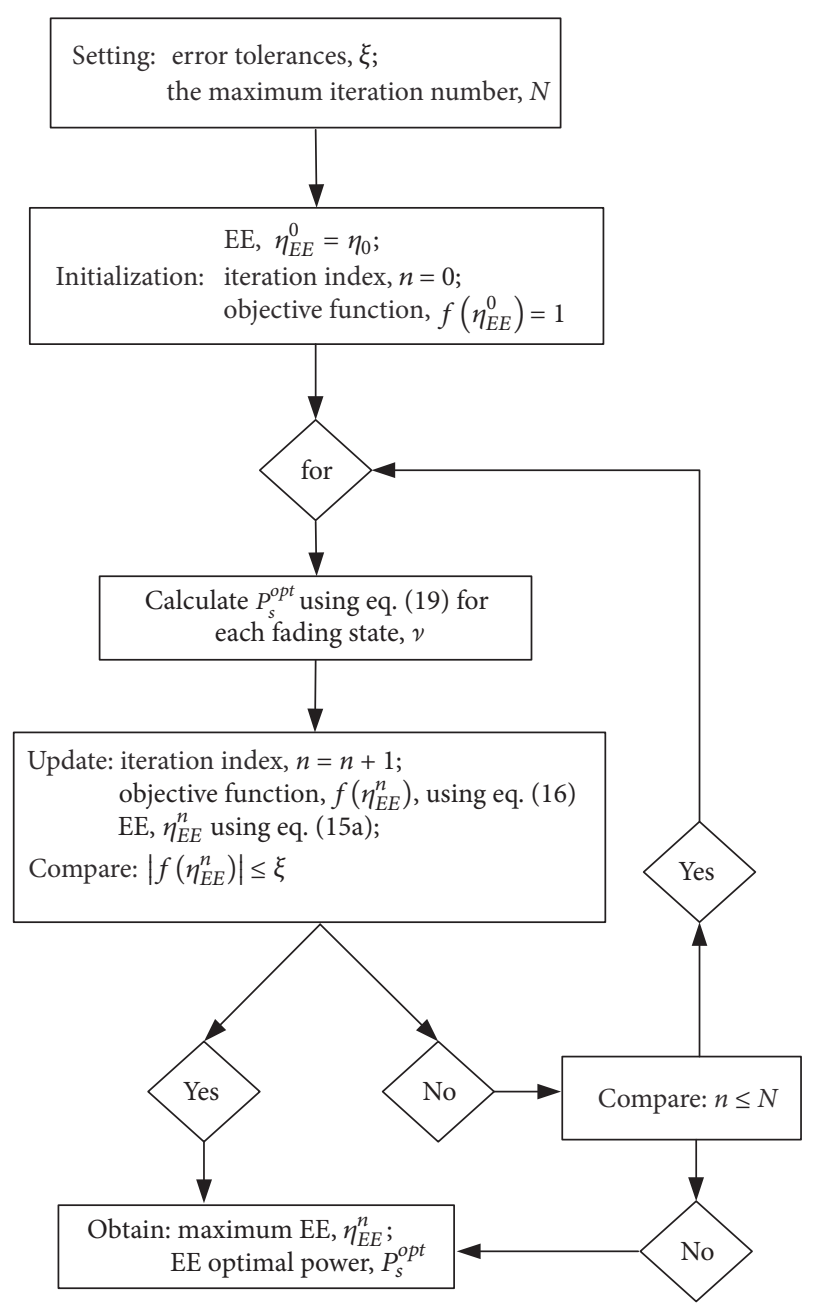

FIGURE 3: Flowchart of Algorithm 2 for EE maximization subject to the PTP constraint and the PIP constraint.

EE and the corresponding optimal power level of the SU are obtained as soon as the optimal power level can enable the fact that $f\left(\eta_{E E}^{n}\right)=0$ holds. Otherwise, an $\xi$-optimal solution with an error tolerance $\xi$ is adopted. If equations $f\left(\eta_{E E}^{n}\right)=0$ and $\left|f\left(\eta_{E E}^{n}\right)\right| \leq \xi$ can not be satisfied, Algorithm 2 stops iterating when the maximum iteration number, $N$, is achieved. The flowchart of Algorithm 2 for EE maximization in delay-insensitive green CR, subject to the PIP constraint and the PTP constraint, is shown in Figure 3.

4.2. EE Maximization in Delay-Sensitive Green CR. In this subsection, the EE maximization problem in delay-sensitive green CR subject to constraint on the PIP and the PTP is studied. In this case, the EE maximization problem in delaysensitive green $\mathrm{CR}$, denoted by problem $\mathbf{P}_{\mathbf{9}}$, can be given as

$$
\begin{aligned}
\mathbf{P}_{\mathbf{9}}: \max _{P_{s}(\nu)} & \eta_{E E}\left(P_{s}(\nu)\right)=\frac{r_{s} \mathbb{E}\left\{1-\chi_{s}(v)\right\}}{\mathbb{E}\left\{\zeta P_{s}(\nu)+P_{C}\right\}} \\
\text { s.t. } & (1) \text { and (20a), (20b) are satisfied }
\end{aligned}
$$


where $\chi_{s}(v)$ is an index associated with the outage event of the $\mathrm{SU}$, given by (14b). Similar to problem $\mathbf{P}_{5}$, problem $\mathbf{P}_{\mathbf{9}}$ can be equivalent to a parameter optimization problem, denoted by problem $\mathbf{P}_{\mathbf{1 0}}$, given as

$$
\begin{array}{rl}
\mathbf{P}_{10}: \max _{P_{s}(\nu) \in S_{3}} & f(\eta) \\
& =r_{s} \mathbb{E}\left\{1-\chi_{s}(v)\right\}-\eta \mathbb{E}\left\{\zeta P_{s}(\nu)+P_{C}\right\}
\end{array}
$$

where $\eta$ is a nonnegative parameter. Similar to problem $\mathbf{P}_{\mathbf{6}}$, problem $\mathbf{P}_{\mathbf{1 0}}$ can be solved by solving the subproblem, given as

$$
\max _{P_{s} \in S_{3}} y\left(P_{s}\right)=-r_{s} \chi_{s}\left(P_{s}\right)-\eta \zeta P_{s}
$$

where $\chi_{s}\left(P_{s}\right)$ is an explicit function of $P_{s} \cdot \chi_{s}\left(P_{s}\right)=1$ for $P_{s}<y$ and $\chi_{s}\left(P_{s}\right)=0$ for $P_{s} \geq y$, and $y$ is given by (18). For a given $\eta$, problem $\mathbf{P}_{10}$ can be solved by solving (27) for all fading states. Let $P_{s}^{o p t}$ denote the optimal power of $\mathbf{P}_{\mathbf{1 0}}$. The following results can be obtained by solving (27). Let $P_{\min }$ denote $P_{\min }=\min \left(r_{s} /(\eta \zeta), P_{I n} / g_{s p}, P_{t h}\right)$.

Case $1\left(y>P_{\min }\right)$. In this case, when $y>r_{s} /(\eta \zeta)$, the minimum power required to guarantee the $\mathrm{OC}$ of the SU is very large and results in very low EE; the SU stops transmitting in order to save energy. Thus, $P_{s}^{o p t}=0$. When $y>P_{I n} / g_{s p}$, the SU that transmits with the minimum power required to guarantee the $\mathrm{OC}$ of the $\mathrm{SU}$ results in unacceptable interference to the PU, and thus the SU stops transmitting in order to protect the PU. Hence, $P_{s}^{o p t}=0$. When $y>P_{\text {th }}$ and the minimum power requiring the guarantee of the OC of the SU is larger than the PTP constraint, the SU stops transmitting in order to protect the SU transmitter. Thus, $P_{s}^{o p t}=0$.

Case $2\left(y \leq P_{\min }\right)$. In this case, $y\left(P_{s}\right)$ achieves the maximum, $-\eta \zeta y$, at $P_{s}=y$. Thus, $P_{s}^{o p t}=y$.

Theorem 9 can be formally stated based on the results obtained above.

Theorem 9. The optimal resource allocation strategy of $\mathbf{P}_{\mathbf{1 0}}$ is given by

$$
\begin{gathered}
P_{s}^{o p t}= \begin{cases}y, & y \leq P_{\min } \\
0, & y>P_{\min }\end{cases} \\
P_{\min }=\min \left(\frac{r_{s}}{\eta \zeta}, \frac{P_{I n}}{g_{s p}}, P_{t h}\right) .
\end{gathered}
$$

Remark 10. In delay-sensitive green CR, under constraints on the PIP and the PTP, the optimal power allocation strategy for maximizing the EE given in Theorem 9 has a similar form to the well-known truncated channel inversion power allocation strategy [32]. The optimal power allocation strategy for green delay-insensitive CR subject to the PIP constraint and the PTP constraint, given in Theorem 9, is equivalent to the optimal power allocation strategy for OP minimization in conventional CR given in $\left[10\right.$, eq. (24)] when $r_{s} /(\eta \zeta) \geq$ $\max \left(P_{\text {In }} / g_{s p}, P_{t h}\right)$. In this case, the maximum EEs achieved by using those two strategies are the same. It is seen that the optimal power allocation strategy for EE maximization in delay-sensitive green $\mathrm{CR}$ relates to the minimum power required to guarantee the $\mathrm{OC}$ of the SU. This outcome is explained by the fact that the SU does not need to increase transmit power when the OC can be guaranteed in delaysensitive green CR.

For a given $\eta$, problem $\mathbf{P}_{10}$ can be efficiently solved by the proposed optimal power allocation strategy given in Theorem 9. In order to solve problem $\mathbf{P}_{\mathbf{9}}$ and obtain the optimal power strategy associated with the achievable maximum EE of the SU, the modified Algorithm 2 can be applied. In this case, the optimal power level, $P_{s}^{o p t}$, is calculated by Theorem 9 . The objective function, $f\left(\eta_{E E}^{n}\right)$, and EE, $\eta_{E E}^{n}$, are calculated by using (26) and (25a), respectively. For brevity, the details are not given here.

4.3. Complexity. As shown in Algorithms 1 and 2, a nonnegative dual variable related to the ATP is required to be updated by using the subgradient method when the ATP constraint is applied, whereas the for-loop is only activated when the PTP constraint is used. Let $N_{1}$ and $N_{2}$ denote the amount of for-loop activation and the number of the iterations required by using the subgradient method of Algorithm 1, respectively. Let $N_{3}$ denote the amount of for-loop activation of Algorithm 2. Let $N_{r}$ denote the number of channel realizations. Let $\ell_{1}$ denote the tolerance error for the subgradient method. Based on the complexity analysis for the subgradient method in [33, $34]$, the total complexity of Algorithm 1 is $\mathrm{O}\left(N_{1} N_{2} N_{r} \times 1 / \ell_{1}^{2}\right)$, whereas the total complexity of Algorithm 2 is $\mathrm{O}\left(N_{3} N_{r}\right)$. It is seen that the complexity of Algorithm 1 is higher than that of Algorithm 2 since it requires obtaining a nonnegative dual variable by using the subgradient method. $N_{2}$ is substantially increased when there are several constraints related to the average metrics, such as ATP. Thus, the decrease of the number of constraints on average metrics can enable the implementation of algorithms with low complexity.

\section{Simulation Results}

In this section, we give simulation results to evaluate the achievable maximum EE of the SU with the proposed optimal power allocation strategies in delay-insensitive green CR and delay-sensitive green CR. The achievable maximum EE with the proposed optimal power allocation strategies is compared with that achieved with the conventional power allocation strategies given in [10]. Simulation results are also presented to evaluate the performance of the two proposed algorithms. The constant circuit power of the SU transmitter and the amplifier coefficient, $P_{C}$ and $\zeta$, are set to be $0.05 W$ and 0.2 . The variance of noise is set as 0.01 . The transmit power of the $\mathrm{PU}, P_{p}$, is set to be $60 \mathrm{~mW}$. The iterative step size of $\tau$ is set as 0.1 . The error tolerances, $\xi$ and $\xi_{1}$, are set as $10^{-4}$. The EE and capacities are evaluated by using $10^{6}$ channel realizations in ergodic fading channels. In all the simulations, the mean values of the channel power gains, $g_{s s}, g_{s p}$, and $h_{p s}$, are set as $2,1.5$, and 1.5. For the Nakagami- $m$ channel, $m$ is chosen as 


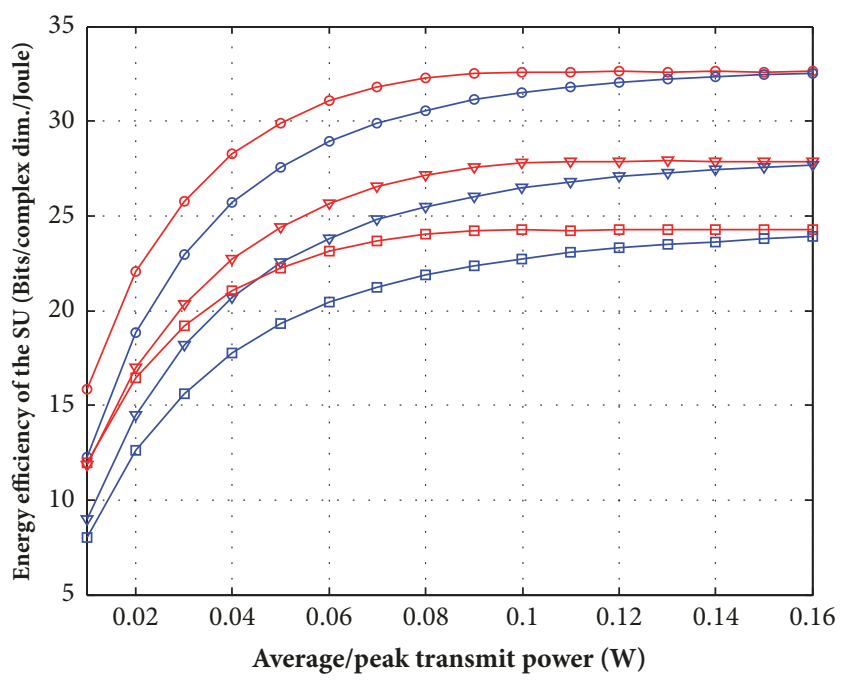

$$
\begin{aligned}
& \rightarrow \text { Aver, } \mathrm{g}_{\mathrm{ss}}: \mathrm{AWGN}, \mathrm{h}_{\mathrm{ps}}: \mathrm{Naka}(\mathrm{m}=0.5), \mathrm{g}_{\mathrm{sp}}: \text { Rayl } \\
& \multimap \text { Peak,gss }: \text { AWGN,h } \mathrm{h}_{\mathrm{ps}}: \mathrm{Naka}(\mathrm{m}=0.5), \mathrm{g}_{\mathrm{sp}}: \text { Rayl } \\
& \rightarrow \text { Aver, } \mathrm{g}_{\mathrm{ss}}: \text { AWGN, } \mathrm{h}_{\mathrm{ps}}: \text { Rayl, } \mathrm{g}_{\mathrm{sp}}: \text { Rayl } \\
& \rightarrow \text { Peak,gss }: \text { AWGN,h } \mathrm{h}_{\mathrm{ps}}: \text { Rayl,g } \mathrm{sp} \text { :Rayl } \\
& \rightarrow \text { Aver,gss }: \text { Rayl, } \mathrm{h}_{\mathrm{ps}}: \text { Rayl,g } \mathrm{g}_{\mathrm{sp}}: \text { Rayl } \\
& \rightarrow \text { Peak,g } \mathrm{g}_{\mathrm{ss}}: \text { Rayl, } \mathrm{h}_{\mathrm{ps}}: \text { Rayl, } \mathrm{g}_{\mathrm{sp}}: \text { Rayl }
\end{aligned}
$$

FIGURE 4: The EE of the SU versus the ATP/PTP constraint for different fading channel models with $P_{I n}=100 \mathrm{~mW}$.

0.5. When the channel is modeled as an AWGN channel, the channel power gain is a constant. In delay-sensitive green $\mathrm{CR}$, the $\mathrm{OC}$ of the $\mathrm{SU}$ is $1 \mathrm{bit} / \mathrm{complex}$ dimension (dim.).

Figure 4 shows the achievable maximum EE of the SU versus the ATP/PTP constraint for different fading channel models in delay-insensitive green CR. The PIP constraint is set as $P_{I n}=100 \mathrm{~mW}$. It is seen that the SU can achieve EE gain under the ATP constraint compared with that achieved under the PTP constraint, irrespective of channel fading models. The reason is that the optimal power allocation strategy can benefit more from a flexibility on the ATP constraint than from that on the PTP constraint. Specifically, a large dynamic range of the transmit power can be obtained under the ATP constraint compared with that achieved under the PTP constraint. As shown in Figure 4, the maximum EE of the SU achieved under the PTP constraint converges to that achieved under the ATP constraint when the ATP/PTP constraint is sufficiently loose compared with the PIP constraint. This can be explained by the fact that the achievable maximum EE of the SU only depends on the PIP constraint when the ATP/PTP constraint is inactive, and the optimal power allocation strategies are the same in this case. This can be seen from Theorems 3 and 7 . The same conclusion has been obtained in [27]. This further verifies our theoretical analysis.

Figure 5 shows the $\mathrm{EE}$ and the $\mathrm{EC}$ of the $\mathrm{SU}$ versus the PIP constraint for the EE maximization or for the conventional EC maximization under the PTP/ATP constraint. The PTP/ATP constraint is set as $P_{t h}=\overline{P_{t h}}=100 \mathrm{~mW}$. All the channels involved are Rayleigh fading. It is seen in Figure 5(a) that the EE of the SU achieved for EE maximization by using the proposed optimal power allocation strategies is not less than that achieved for EC maximization by using the power allocation strategies proposed in [10]. This indicates that the proposed optimal power allocation strategies for EE maximization can guarantee that the SU obtains the maximum value of $\mathrm{EE}$, whereas the optimal power allocation strategies proposed in [10] for EC maximization can not do that. Thus, those strategies proposed in [10] are not appropriate in delay-insensitive green $\mathrm{CR}$ in terms of $\mathrm{EE}$ maximization. It is interesting to note in Figure 5(a) that the $\mathrm{EE}$ of the SU achieved by using the conventional optimal power allocation strategy under the PTP constraint may be higher than that achieved by using the conventional optimal power allocation strategy under the ATP constraint. The reason is that the conventional optimal power allocation strategies for EC maximization are not optimal with respect to EE maximization in delay-insensitive green CR. However, it is seen in Figure 5(b) that the EC of the SU achieved by using the conventional optimal power allocation strategy under the PTP constraint is always smaller than that achieved by using the conventional optimal power allocation strategy under the ATP constraint. Similar results have been obtained for $\mathrm{CR}$ under spectrum sharing for EC maximization in $[10,14]$.

Figure 6 offers a deep insight into the maximum EE of the SU versus the PIP constraint and the ATP/PTP constraint in delay-insensitive green CR. All the channels involved are Rayleigh fading channels. It is seen that the maximum EE of the SU increases with the loose PIP constraint or with the loose ATP/PTP constraint. This is explained by the fact that the optimal power level increases with the looser constraints. It is also seen that the maximum EE of the SU achieved under the ATP constraint is larger than that achieved under the PTP constraint.

Figure 7 shows the EE of the SU versus the ATP/PTP constraint for different fading channel models in delaysensitive green CR. The PIP constraint is set as $P_{\text {In }}=$ $100 \mathrm{~mW}$. In delay-sensitive green CR, it is also seen that the EE of the SU achieved for EE maximization under the ATP constraint is larger than that achieved for EE maximization under the PTP constraint, irrespective of channel fading models. This can also be explained by the fact that the optimal power allocation strategy is more flexible under the ATP constraint than the optimal power allocation strategy under the PTP constraint.

Figure 8 presents a comparison of the EE and the OP of the SU achieved for the EE maximization with that achieved for the conventional OP minimization in delay-sensitive green CR. The PTP/ATP constraint is set as $P_{t h}=\overline{P_{t h}}=$ $50 \mathrm{~mW}$. All the involved channels are Rayleigh fading. It is seen that the maximum EE of the SU for EE maximization by using the proposed optimal power allocation strategy under the ATP constraint is not less than that of the SU for OP minimization by using the optimal power allocation strategy given in [10, eq. (28)]. This indicates that the conventional optimal power allocation strategy for OP minimization given in $[10$, eq. (28)] is not always optimal in delay-sensitive green 


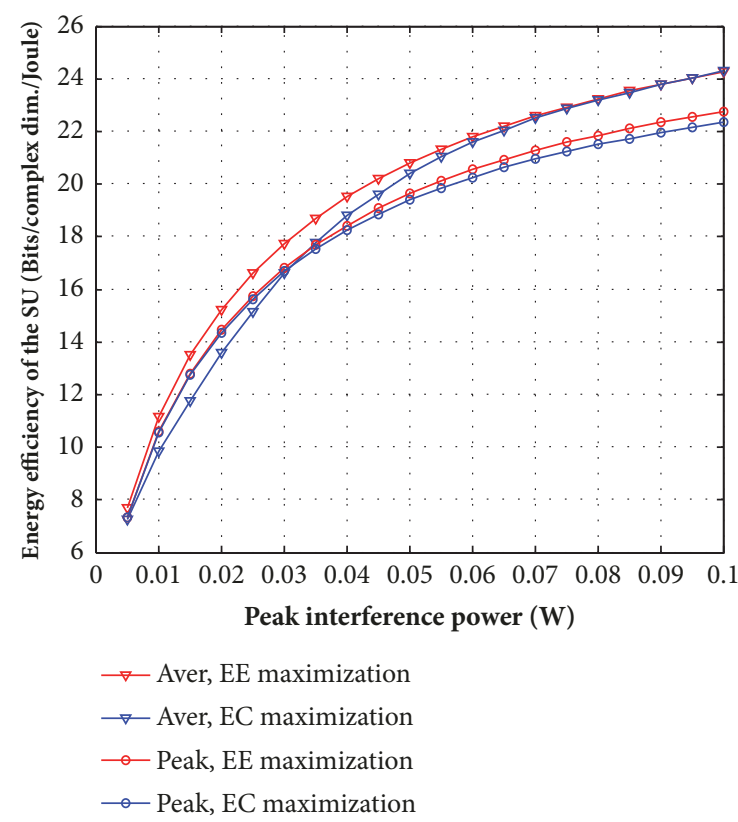

(a)

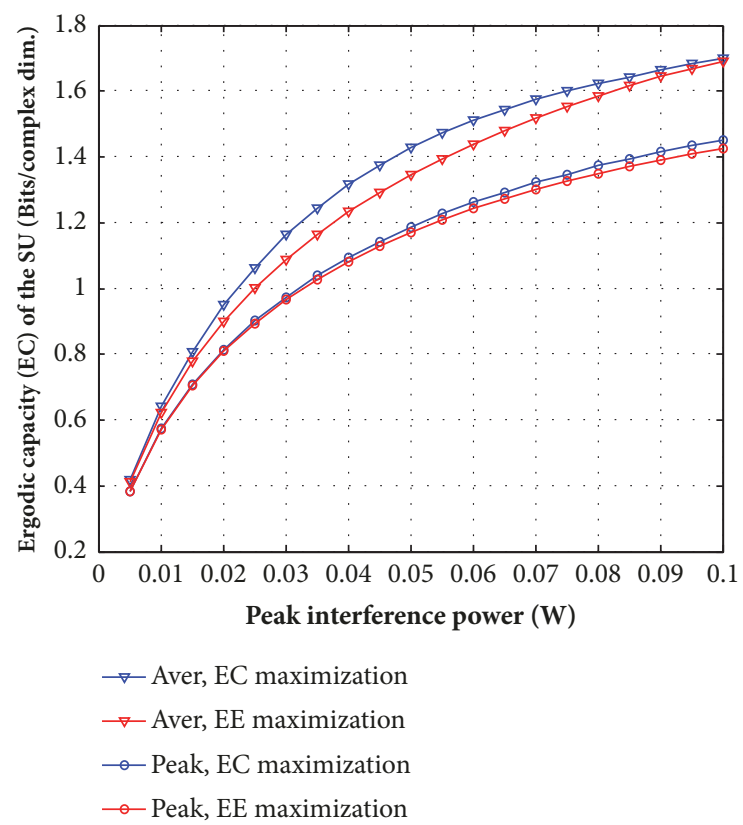

(b)

FIgURE 5: (a) The EE of the SU versus the PIP constraint for EE maximization or EC maximization under the PTP/ATP constraint, $P_{t h}=$ $\overline{P_{t h}}=100 \mathrm{~mW}$. (b) The EC of the SU versus the PIP constraint for EE maximization or EC maximization under the PTP/ATP constraint, $P_{t h}=\overline{P_{t h}}=100 \mathrm{~mW}$.

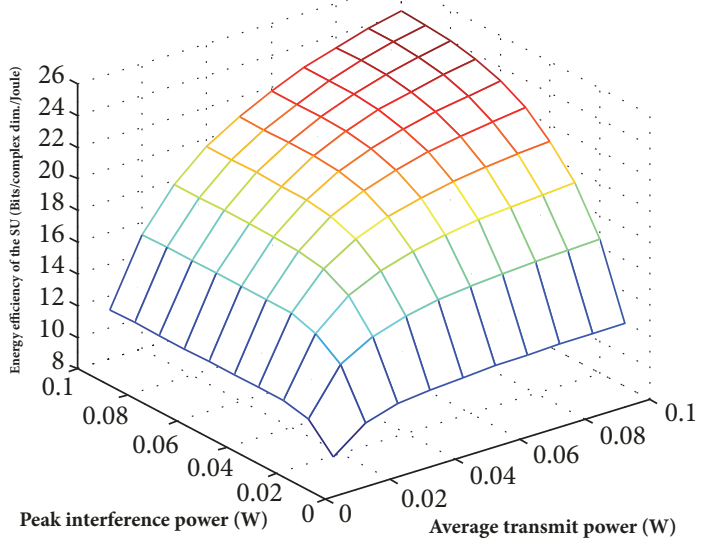

(a)

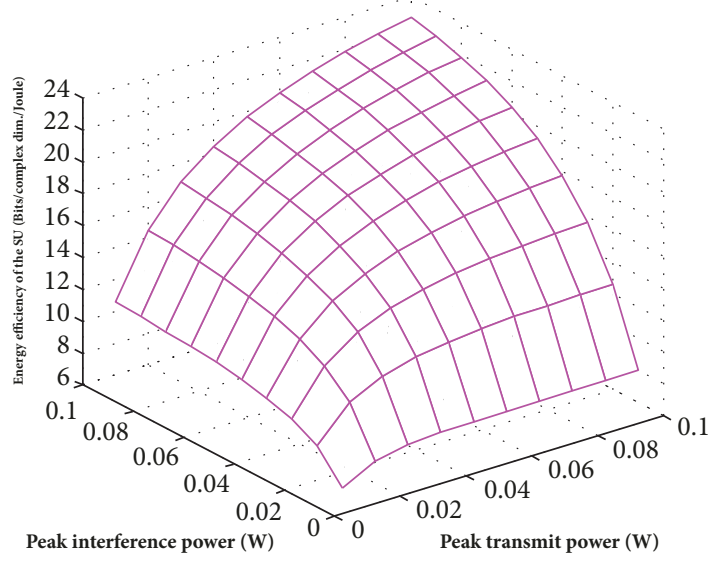

(b)

FIgure 6: (a) The EE of the SU versus the PIP constraint and the ATP constraint in delay-insensitive green CR. (b) The EE of the SU versus the PIP constraint and the PTP constraint in delay-insensitive green CR.

CR in terms of EE maximization. As shown in Figure 8(a), when the PIP constraint is sufficiently tense compared with the ATP constraint, i.e., $P_{\text {In }}=30 \mathrm{~mW}$, the EE achieved by using the proposed optimal power allocation strategy is equal to that achieved by using the conventional optimal power allocation strategy. The reason is that only the PIP constraint is active and those two optimal strategies are equivalent in this case. This is consistent with our theoretical analysis given in Section 3.2. It is seen that the EE of the SU achieved by using the proposed optimal power allocation strategy for EE maximization given in Theorem 9 and that achieved by using the optimal power allocation strategy for OP minimization given in [10, eq. (24)] are the same when the PIP constraint and the PTP constraint are very tense. This is explained by the fact that the proposed optimal power allocation strategy given in Theorem 9 and the optimal power allocation strategy given in [10, eq. (24)] are equivalent when the EE of the SU is small and the equation $r_{s} /(\eta \zeta) \geq \max \left(P_{I n} / g_{s p}, P_{t h}\right)$ always holds. The results confirm our theoretical analysis given in Section 4.2.

Figure 9 is presented to show that the optimal power allocation strategy for OP minimization proposed in $[10$, eq. 


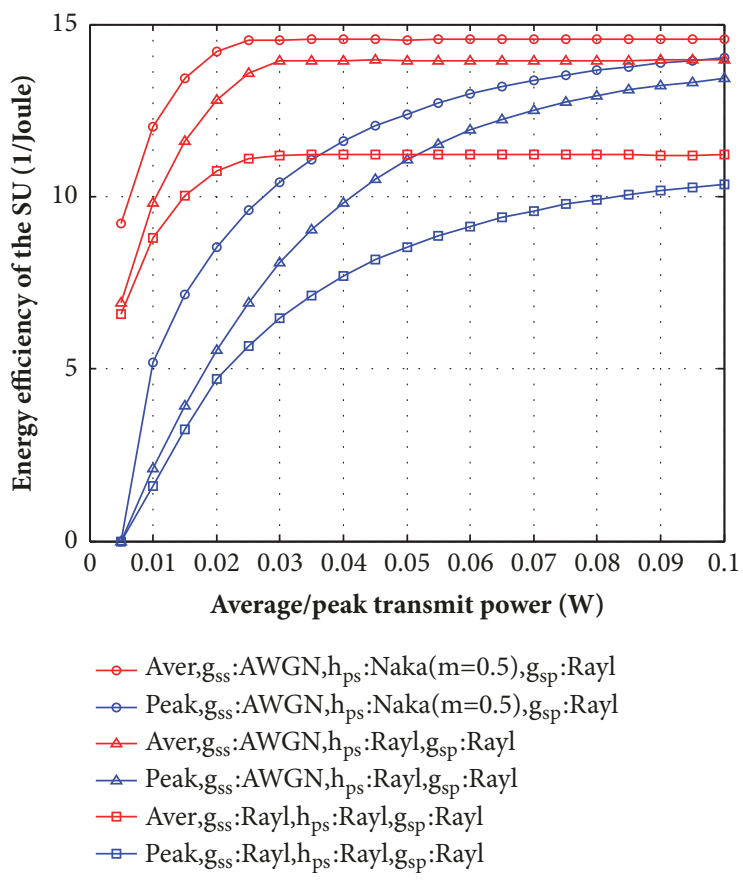

FIgURE 7: The EE of the SU versus the ATP/PTP constraint for different fading channel models with $P_{I n}=100 \mathrm{~mW}$ in delay-sensitive green CR.
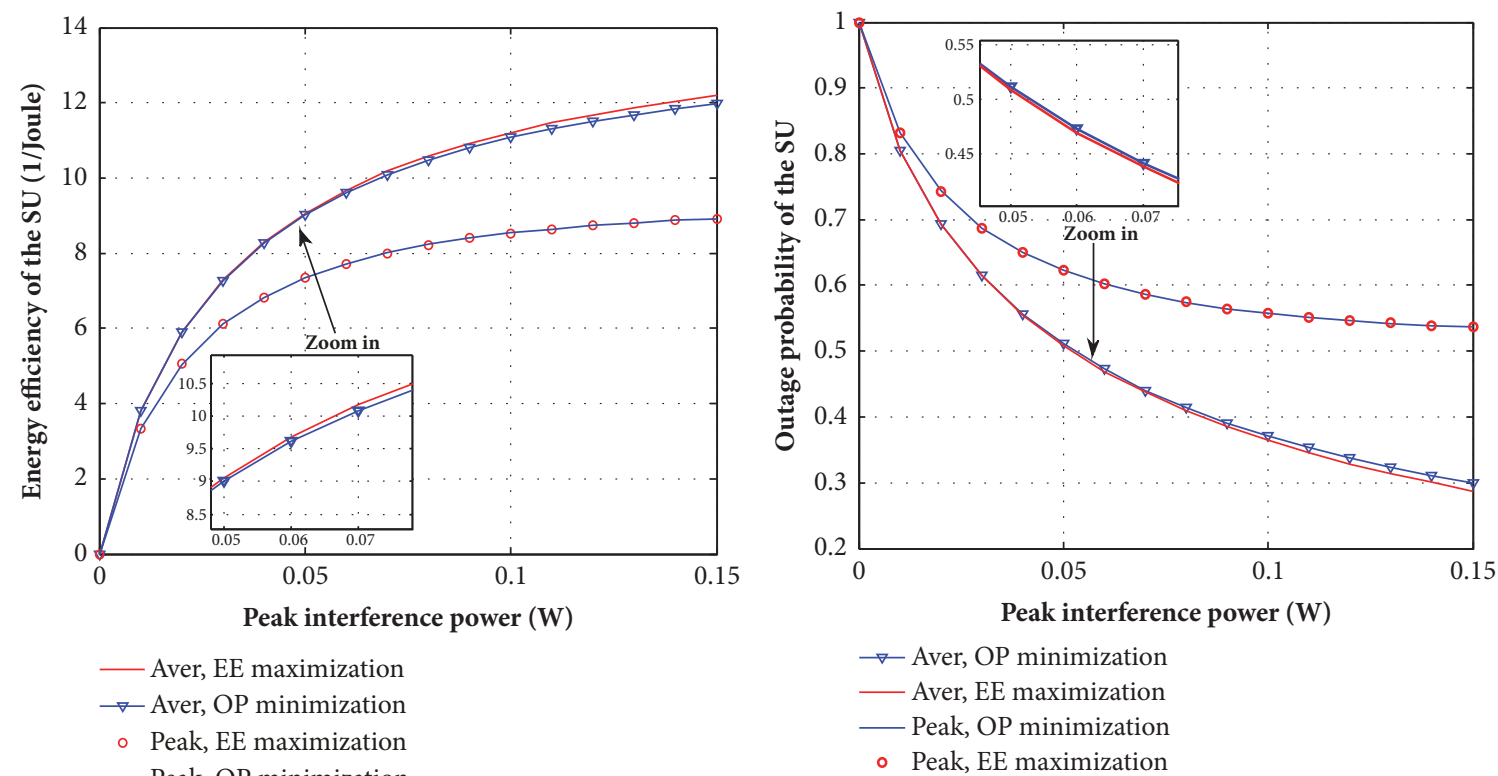

(b)

FIgURE 8: (a) The EE of the SU versus the PIP constraint for EE maximization or OP minimization under the PTP/ATP constraint in delaysensitive green CR, $P_{t h}=\overline{P_{t h}}=50 \mathrm{~mW}$. (b) The OP of the SU versus the PIP constraint for EE maximization or OP minimization under the $\mathrm{PTP} / \mathrm{ATP}$ constraint in delay-sensitive green $\mathrm{CR}, P_{t h}=\overline{P_{t h}}=50 \mathrm{~mW}$.

(24)] is not always optimal in delay-sensitive green CR while the proposed power allocation strategy given in Theorem 9 is optimal with respect to EE maximization. Since those two strategies are equivalent when the PIP constraint and the PTP constraint are very tense, as shown in Figure 8, another green $\mathrm{CR}$ is considered. In this green $\mathrm{CR}$, the transmit power of the PU is set as $P_{p}=500 \mathrm{~mW}$. The PTP constraint is $P_{t h}=2 \mathrm{~W}$. The channel gains, $g_{s p}$, and $h_{p s}$, model Rayleigh fading channels with mean 1.5. It is seen that the EE of the SU achieved for EE maximization by using the proposed power strategy given in Theorem 9 is larger than that achieved for OP minimization by using the power allocation strategy 
TABLE 1: The comparison of time (s) taken by Algorithm 1 with time taken by Algorithm 2.

\begin{tabular}{lccccccccc}
\hline \multirow{2}{*}{ Green CR } & & \multicolumn{9}{c}{ Transmit power } \\
& & $20 \mathrm{~mW}$ & $30 \mathrm{~mW}$ & $40 \mathrm{~mW}$ & $50 \mathrm{~mW}$ & $60 \mathrm{~mW}$ & $70 \mathrm{~mW}$ & $80 \mathrm{~mW}$ & $90 \mathrm{~mW}$ \\
\hline \multirow{2}{*}{ Delay-insensitive } & Average & 535.162 & 313.368 & 212.008 & 155.220 & 119.644 & 95.025 & 77.328 \\
& Peak & 0.198 & 0.203 & 0.199 & 0.199 & 0.208 & 0.200 & 0.199 & 0.198 \\
\hline \multirow{2}{*}{ Delay-sensitive } & Average & 856.223 & 733.604 & 242.051 & 198.239 & 130.344 & 81.732 & 61.740 & 50.934 \\
& Peak & 0.170 & 0.168 & 0.182 & 0.177 & 0.177 & 0.180 & 0.175 & 0.177 \\
\hline
\end{tabular}

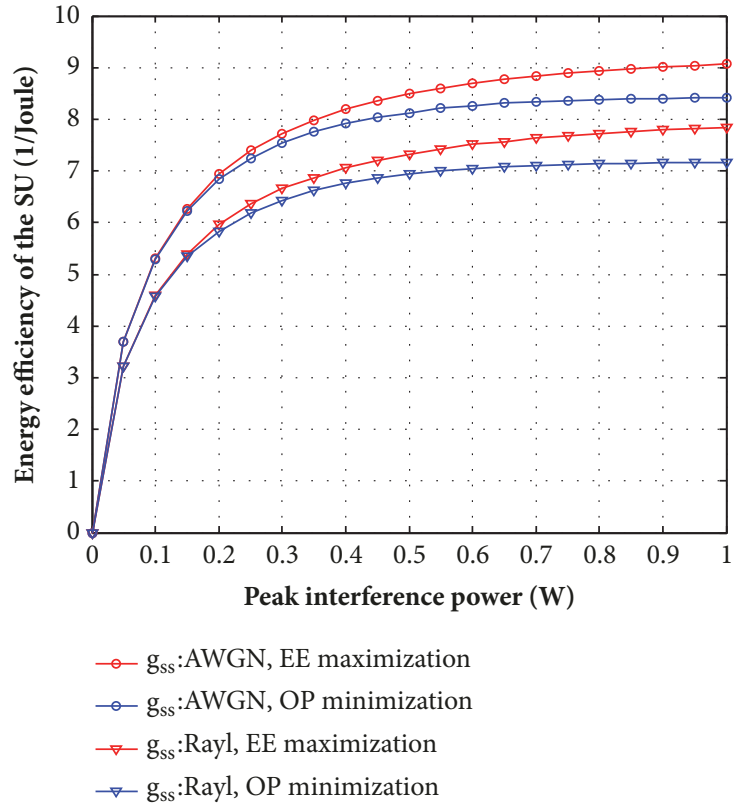

FIGURE 9: The EE of the SU versus the PTP constraint for EE maximization or OP minimization under the PTP constraint with $P_{t h}=2 W$.

proposed in [10, eq. (24)] when the PIP is loose. It is also seen that the EE of the SU when $g_{s s}$ models the AWGN channel is larger than that when $g_{s s}$ models the Rayleigh channel. This also indicates that the fading of the channel between the SUTx and the SU-Rx is unfavorable to the SU in terms of EE maximization.

Table 1 shows comparisons of time taken by Algorithm 1 with the ATP constraint to time taken by Algorithm 2 with the PTP constraint in delay-insensitive green CR and delaysensitive green CR. The unit of time is $s$. The PIP constraint is set as $P_{\text {In }}=0.15 \mathrm{~W}$. All the channels involved are Rayleigh fading. Note that Algorithm 1 is proposed to solve the EE maximization problem under the ATP constraint, whereas Algorithm 2 is given for solving the EE maximization problem under the PTP constraint. It is seen that the time spent on solving the EE maximization problem under the ATP constraint is much more than the time spent on solving the EE maximization problem under the PTP constraint, irrespective of whether the case is delay-insensitive green CR or delay-sensitive green CR. The reason is that much time is spent on computing the nonnegative dual variable with respect to the ATP constraint. Thus, a green CR with the PTP constraint can be implemented with low complexity compared with the green CR with the ATP constraint in terms of EE maximization. On the other hand, the SU can achieve EE gain under the ATP constraint in contrast with the PTP constraint. Thus, the design of a green CR should take the tradeoff between its complexity and its maximum EE into consideration.

\section{Conclusion}

Energy efficiency maximization problems were studied in delay-insensitive green CR and delay-sensitive green CR. Optimal power allocation strategies for delay-insensitive green $\mathrm{CR}$ and delay-sensitive green $\mathrm{CR}$ were designed to maximize the achievable EE of the SU. Two algorithms based on the proposed optimal resource allocation strategies were proposed. It is shown that $\mathrm{CR}$ with the instantaneous metric constraint can achieve implementation with low complexity in contrast with CR with the average metric constraint. Simulation results illustrated that the SU can achieve EE gain under the ATP constraint compared with that achieved under the PTP constraint in terms of EE maximization. The design of a green CR system should take the tradeoff between its complexity and its achievable maximum EE into consideration.

\section{Appendix}

\section{A. Proof of Theorem 1}

A strictly quasiconcave function is defined as follows. Let $S$ be a nonempty convex set. $f$ is a strictly quasiconcave function if, for each $x_{1}, x_{2} \in S$ with $f\left(x_{1}\right) \neq f\left(x_{2}\right)$, one has

$$
\begin{aligned}
f\left[\lambda x_{1}+(1-\lambda) x_{2}\right]>\min \left\{f\left(x_{1}\right),\right. & \left.f\left(x_{2}\right)\right\} \\
& \text { for } \lambda \in(0,1) .
\end{aligned}
$$

Let $R_{E E}^{E C}\left(P_{s}\right)$ and $P_{E E}\left(P_{s}\right)$ denote the numerator and the denominator of $\eta_{E E}\left(P_{s}(\nu)\right)$, respectively; namely, $R_{E E}^{E C}\left(P_{s}\right)=$ $\mathbb{E}\left\{\log _{2}\left(1+g_{s s} P_{s} /\left(h_{p s} P_{p}+\sigma_{w}^{2}\right)\right)\right\}$ and $P_{E E}\left(P_{s}\right)=\mathbb{E}\left\{\zeta P_{s}+\right.$ $\left.P_{C}\right\} . R_{E E}^{E C}\left(P_{s}\right)$ is a concave function of $P_{s}$ since $\log _{2}(1+$ $\left.g_{s s} P_{s} /\left(h_{p s} P_{p}+\sigma_{w}^{2}\right)\right)$ is a concave function of $P_{s}$ [31]. $P_{E E}\left(P_{s}\right)$ is a convex function of $P_{s}$ and also is a concave function of $P_{s}$ since $\zeta P_{s}+P_{C}$ is an affine function of $P_{s}$. Note that $P_{E E}\left(P_{s}\right)>0$. For brevity, the index $v$ for the fading state is dropped. Let $P_{s}^{1}, P_{s}^{2} \in S_{1}$ and $0 \leq \lambda \leq 1$. It is assumed that 
$\eta_{E E}\left(P_{s}^{1}\right)<\eta_{E E}\left(P_{s}^{2}\right)$. Now, since $R_{E E}^{E C}\left(P_{s}\right)$ is a strictly concave function of $P_{s}$, one has

$$
\begin{aligned}
& R_{E E}^{E C}\left[\lambda P_{s}^{1}+(1-\lambda) P_{s}^{2}\right] \\
& >\lambda R_{E E}^{E C}\left(P_{s}^{1}\right)+(1-\lambda) R_{E E}^{E C}\left(P_{s}^{2}\right) \\
& >\lambda R_{E E}^{E C}\left(P_{s}^{1}\right)+(1-\lambda) \frac{R_{E E}^{E C}\left(P_{s}^{1}\right)}{P_{E E}\left(P_{s}^{1}\right)} P_{E E}\left(P_{s}^{2}\right) \\
& =\frac{R_{E E}^{E C}\left(P_{s}^{1}\right)}{P_{E E}\left(P_{s}^{1}\right)}\left[\lambda P_{E E}\left(P_{s}^{1}\right)+(1-\lambda) P_{E E}\left(P_{s}^{2}\right)\right] .
\end{aligned}
$$

Since $\lambda P_{E E}\left(P_{s}^{1}\right)+(1-\lambda) P_{E E}\left(P_{s}^{2}\right)=P_{E E}\left[\lambda P_{s}^{1}+(1-\lambda) P_{s}^{2}\right]$, one has

$$
\frac{R_{E E}^{E C}\left[\lambda P_{s}^{1}+(1-\lambda) P_{s}^{2}\right]}{P_{E E}\left[\lambda P_{s}^{1}+(1-\lambda) P_{s}^{2}\right]}>\frac{R_{E E}^{E C}\left(P_{s}^{1}\right)}{P_{E E}\left(P_{s}^{1}\right)} .
$$

Thus, $\eta_{E E}\left(P_{s}(\nu)\right)$ is strictly quasiconcave on $S_{1}$.

On the other hand, since $R_{E E}^{E C}\left(P_{s}\right)$ and $P_{E E}\left(P_{s}\right)$ are differentiable, the derivation of $\eta_{E E}\left(P_{s}\right)$ can be given as

$$
\begin{aligned}
& \frac{d \eta_{E E}\left(P_{s}\right)}{d P_{s}} \\
& =\frac{1}{P_{E E}^{2}\left(P_{s}\right)} \\
& \quad \times\left[\frac{d R_{E E}^{E C}\left(P_{s}\right)}{d P_{s}} P_{E E}\left(P_{s}\right)-\frac{d P_{E E}\left(P_{s}\right)}{d P_{s}} R_{E E}^{E C}\left(P_{s}\right)\right] .
\end{aligned}
$$

Let $P_{v}$ denote the value of $P_{s}$ that makes the derivative of $\eta_{E E}\left(P_{s}\right)$ be equal to zero. One has

$$
\frac{d R_{E E}^{E C}\left(P_{v}\right)}{d P_{v}}=\frac{d P_{E E}\left(P_{v}\right)}{d P_{v}} \eta_{E E}\left(P_{v}\right) .
$$

Since $R_{E E}^{E C}\left(P_{s}\right)$ is a concave function of $P_{s}$, one has

$$
\begin{aligned}
R_{E E}^{E C}\left(P_{s}\right) & \leq R_{E E}^{E C}\left(P_{v}\right)+\frac{d R_{E E}^{E C}\left(P_{v}\right)}{d P_{v}}\left(P_{s}-P_{v}\right) \\
& =R_{E E}^{E C}\left(P_{v}\right)+\frac{d P_{E E}\left(P_{v}\right)}{d P_{v}} \eta_{E E}\left(P_{v}\right)\left(P_{s}-P_{v}\right) .
\end{aligned}
$$

Since $P_{E E}\left(P_{s}\right)$ is a convex function of $P_{s}$ and also is a concave function of $P_{s}$, one has

$$
\frac{d P_{E E}\left(P_{v}\right)}{d P_{v}}\left(P_{s}-P_{v}\right)=P_{E E}\left(P_{s}\right)-P_{E E}\left(P_{v}\right) \text {. }
$$

According to (A.6) and (A.7), one has

$$
\frac{R_{E E}^{E C}\left(P_{s}\right)}{P_{s}\left(P_{s}\right)} \leq \eta_{E E}\left(P_{v}\right) \text {. }
$$

It is seen that $P_{v}$ is the local maximum of $\eta_{E E}\left(P_{s}\right)$. Thus, $\eta_{E E}\left(P_{s}\right)$ is strictly pseudoconcave since $\eta_{E E}\left(P_{s}\right)$ is strictly quasiconcave and $R_{E E}^{E C}\left(P_{s}\right)$ is a concave function. Moreover, $\eta_{E E}\left(P_{s}\right) \leq \eta_{E E}\left(P_{v}\right)$ holds at any $P_{s}$ if $d \eta_{E E}\left(P_{v}\right) / d P_{v}=0$. Thus, $P_{v}$ is the global maximum of $\eta_{E E}\left(P_{s}\right)$. The proof is complete.

\section{B. Proof of Theorem 2}

Let $P_{s}^{o p t}(\nu)$ and $\eta_{o p t}\left(P_{s}^{o p t}(\nu)\right)$ denote the optimal solution of $\mathbf{P}_{\mathbf{1}}$ and the corresponding maximum value of EE. One has

$$
\begin{aligned}
\eta_{o p t}\left(P_{s}^{o p t}(\nu)\right)=\max _{P_{s}(\nu) \in S_{1}} \\
\cdot \frac{\mathbb{E}\left\{\log _{2}\left(1+g_{s s}(\nu) P_{s}(\nu) /\left(h_{p s}(\nu) P_{p}+\sigma_{w}^{2}\right)\right)\right\}}{\mathbb{E}\left\{\zeta P_{s}(\nu)+P_{C}\right\}} \\
=\frac{\mathbb{E}\left\{\log _{2}\left(1+g_{s s}(\nu) P_{s}^{o p t}(\nu) /\left(h_{p s}(\nu) P_{p}+\sigma_{w}^{2}\right)\right)\right\}}{\mathbb{E}\left\{\zeta P_{s}^{o p t}(\nu)+P_{C}\right\}} .
\end{aligned}
$$

Thus, one has

$$
\begin{aligned}
& \frac{\mathbb{E}\left\{\log _{2}\left(1+g_{s s}(\nu) P_{s}(\nu) /\left(h_{p s}(\nu) P_{p}+\sigma_{w}^{2}\right)\right)\right\}}{\mathbb{E}\left\{\zeta P_{s}(\nu)+P_{C}\right\}} \\
& \leq \frac{\mathbb{E}\left\{\log _{2}\left(1+g_{s s}(\nu) P_{s}^{o p t}(\nu) /\left(h_{p s}(\nu) P_{p}+\sigma_{w}^{2}\right)\right)\right\}}{\mathbb{E}\left\{\zeta P_{s}^{o p t}(\nu)+P_{C}\right\}} \\
& =\eta_{o p t}\left(P_{s}^{o p t}(\nu)\right) \\
& \mathbb{E}\left\{\log _{2}\left(1+\frac{g_{s s}(\nu) P_{s}(\nu)}{h_{p s}(\nu) P_{p}+\sigma_{w}^{2}}\right)\right\} \\
& -\eta_{\text {opt }}\left(P_{s}^{o p t}(\nu)\right) \mathbb{E}\left\{\zeta P_{s}(\nu)+P_{C}\right\} \leq 0 \\
& \mathbb{E}\left\{\log _{2}\left(1+\frac{g_{s s}(\nu) P_{s}^{o p t}(\nu)}{h_{p s}(\nu) P_{p}+\sigma_{w}^{2}}\right)\right\} \\
& -\eta_{o p t}\left(P_{s}^{o p t}(\nu)\right) \mathbb{E}\left\{\zeta P_{s}^{o p t}(\nu)+P_{C}\right\}=0 .
\end{aligned}
$$

According to (B.2a), (B.2b), and (B.2c), the maximum of $f(\eta)$ is zero and is achieved when the optimal EE is obtained and the optimal power is adopted. It is proved that the optimized solution of $\mathbf{P}_{\mathbf{1}}$ is the optimization of $\mathbf{P}_{\mathbf{2}}$. On the other hand, let $P^{\text {opt }}$ denote the optimized solution of the problem, given as

$$
\begin{aligned}
\max _{P_{s}(\nu) \in S_{1}} f\left(\eta_{o p t}\right) \\
=\mathbb{E}\left\{\log _{2}\left(1+\frac{g_{s s}(\nu) P_{s}(\nu)}{h_{p s}(\nu) P_{p}+\sigma_{w}^{2}}\right)\right\} \\
-\eta_{o p t} \mathbb{E}\left\{\zeta P_{s}(\nu)+P_{C}\right\}=0 .
\end{aligned}
$$

One has

$$
\begin{aligned}
\mathbb{E} & \left\{\log _{2}\left(1+\frac{g_{s s}(\nu) P_{s}(\nu)}{h_{p s}(\nu) P_{p}+\sigma_{w}^{2}}\right)\right\} \\
& -\eta_{o p t} \mathbb{E}\left\{\zeta P_{s}(\nu)+P_{C}\right\} \\
& \leq \mathbb{E}\left\{\log _{2}\left(1+\frac{g_{s s}(\nu) P^{o p t}(\nu)}{h_{p s}(\nu) P_{p}+\sigma_{w}^{2}}\right)\right\} \\
& -\eta_{o p t} \mathbb{E}\left\{\zeta P^{o p t}(\nu)+P_{C}\right\}
\end{aligned}
$$




$$
\begin{aligned}
& \mathbb{E}\left\{\log _{2}\left(1+\frac{g_{s s}(\nu) P^{o p t}(\nu)}{h_{p s}(\nu) P_{p}+\sigma_{w}^{2}}\right)\right\} \\
& -\eta_{o p t} \mathbb{E}\left\{\zeta P^{o p t}(\nu)+P_{C}\right\}=0 \\
& \frac{\mathbb{E}\left\{\log _{2}\left(1+g_{s s}(v) P_{s}(v) /\left(h_{p s}(\nu) P_{p}+\sigma_{w}^{2}\right)\right)\right\}}{\mathbb{E}\left\{\zeta P_{s}(\nu)+P_{C}\right\}} \\
& \quad \leq \eta_{o p t} .
\end{aligned}
$$

Thus, it is seen that the optimized solution of $\mathbf{P}_{2}$ is also the optimized solution of $\mathbf{P}_{\mathbf{1}}$. The proof is complete.

\section{Data Availability}

The underlying data is not provided since it can be easily obtained by using the algorithms proposed in this article.

\section{Conflicts of Interest}

The authors declare that they have no conflicts of interest.

\section{References}

[1] G. Y. Li, Z. Xu, C. Xiong et al., "Energy-efficient wireless communications: tutorial, survey, and open issues," IEEE Wireless Communications Magazine, vol. 18, no. 6, pp. 28-35, 2011.

[2] F. Zhou, Y. Wu, R. Q. Hu, Y. Wang, and K. K. Wong, "Energyefficient NOMA enabled heterogeneous cloud radio access networks," IEEE Network, vol. 32, no. 2, pp. 152-160, 2018.

[3] Q. Wu, G. Y. Li, W. Chen, D. W. K. Ng, and R. Schober, "An overview of sustainable green $5 \mathrm{G}$ networks," IEEE Wireless Communications Magazine, vol. 24, no. 4, pp. 72-80, 2017.

[4] F. Zhou, Z. Chu, H. Sun, R. Q. Hu, and L. Hanzo, "Artificial noise aided secure cognitive beamforming for cooperative MISO-NOMA using SWIPT,' IEEE Journal on Selected Areas in Communications, 2018.

[5] E. Z. Tragos, S. Zeadally, A. G. Fragkiadakis, and V. A. Siris, "Spectrum assignment in cognitive radio networks: a comprehensive survey," IEEE Communications Surveys \& Tutorials, vol. 15, no. 3, pp. 1108-1135, 2013.

[6] Z. Chu, F. Zhou, Z. Zhu, R. Q. Hu, and P. Xiao, "Wireless powered sensor networks for internet of things: maximum throughput and optimal power allocation," IEEE Internet of Things Journal, vol. 5, no. 1, pp. 310-321, 2018.

[7] F. Zhou, Y. Wu, Y. Liang, Z. Li, Y. Wang, and K. Wong, "State of the art, taxonomy, and open issues on NOMA in cognitive radio networks," IEEE Wireless Communications Magazine, In press.

[8] S. Zhang, Q. Wu, S. Xu, and G. Y. Li, "Fundamental green tradeoffs: progresses, challenges, and impacts on $5 \mathrm{G}$ networks," IEEE Communications Surveys \& Tutorials, vol. 19, no. 1, pp. 3356, 2017.

[9] R. Zhang, "On peak versus average interference power constraints for protecting primary users in cognitive radio networks," IEEE Transactions on Wireless Communications, vol. 8, no. 4, pp. 2112-2120, 2009.

[10] X. Kang, Y. C. Liang, H. K. Garg, and R. Zhang, "Optimal power allocation for fading channels in cognitive radio networks: ergodic capacity and outage capacity," IEEE Transactions on Wireless Communications, vol. 8, no. 2, pp. 940-950, 2009.
[11] X. Kang, R. Zhang, Y. Liang, and H. K. Garg, "Optimal power allocation strategies for fading cognitive radio channels with primary user outage constraint," IEEE Journal on Selected Areas in Communications, vol. 29, no. 2, pp. 374-383, 2011.

[12] X. Gong, A. Ispas, G. Dartmann, and G. Ascheid, "Power allocation and performance analysis in spectrum sharing systems with statistical CSI," IEEE Transactions on Wireless Communications, vol. 12, no. 4, pp. 1819-1831, 2013.

[13] X. Gong, A. Ispas, G. Dartmann, and G. Ascheid, "Outageconstrained power allocation in spectrum sharing systems with partial CSI," IEEE Transactions on Communications, vol. 62, no. 2, pp. 452-466, 2014.

[14] L. Musavian and S. Aissa, "Capacity and power allocation for spectrum-sharing communications in fading channels," IEEE Transactions on Wireless Communications, vol. 8, no. 1, pp. 148156, 2009.

[15] Y. He and S. Dey, "Power allocation for secondary outage minimization in spectrum sharing networks with limited feedback," IEEE Transactions on Communications, vol. 61, no. 7, pp. 26482663, 2013.

[16] W.-C. Pao and Y.-F. Chen, "Adaptive gradient-based methods for adaptive power allocation in OFDM-based cognitive radio networks," IEEE Transactions on Vehicular Technology, vol. 63, no. 2, pp. 836-848, 2014.

[17] G. Ozcan and M. C. Gursoy, "Optimal power control for underlay cognitive radio systems with arbitrary input distributions," IEEE Transactions on Wireless Communications, vol. 14, no. 8, pp. 4219-4233, 2015.

[18] S. Gong, P. Wang, and L. Duan, "Distributed power control with robust protection for PUs in cognitive radio networks," IEEE Transactions on Wireless Communications, vol. 14, no. 6, pp. 3247-3258, 2015.

[19] Y. Wang, W. Xu, K. Yang, and J. Lin, "Optimal energy-efficient power allocation for OFDM-based cognitive radio networks," IEEE Communications Letters, vol. 16, no. 9, pp. 1420-1423, 2012.

[20] J. Mao, G. Xie, J. Gao, and Y. Liu, "Energy efficiency optimization for ofdm-based cognitive radio systems: a water-filling factor aided search method," IEEE Transactions on Wireless Communications, vol. 12, no. 5, pp. 2366-2375, 2013.

[21] C. Luo, G. Min, F. R. Yu, M. Chen, L. T. Yang, and V. C. M. Leung, "Energy-efficient distributed relay and power control in cognitive radio cooperative communications," IEEE Journal on Selected Areas in Communications, vol. 31, no. 11, pp. 2442-2452, 2013.

[22] A. Alabbasi, Z. Rezki, and B. Shihada, "Energy efficient resource allocation for cognitive radios: A generalized sensing analysis," IEEE Transactions on Wireless Communications, vol. 14, no. 5, pp. 2455-2469, 2015.

[23] G. Ozcan and M. C. Gursoy, "Energy-efficient power adaptation for cognitive radio systems under imperfect channel sensing," in Proceedings of the IEEE Conference on Computer Communications Workshops (INFOCOM '14), pp. 706-711, IEEE, Ontario, Canada, April-May 2014.

[24] F. Zhou, Y. Wang, D. Qin, Y. Wang, and Y. Wu, "Secure EE maximisation in green CR: guaranteed SC," IET Communications, vol. 11, no. 16, pp. 2507-2513, 2017.

[25] L. Wang, M. Sheng, X. Wang, Y. Zhang, and X. Ma, "Mean energy efficiency maximization in cognitive radio channels with PU outage constraint," IEEE Communications Letters, vol. 19, no. 2, pp. 287-290, 2015. 
[26] Q. Wu, G. Y. Li, W. Chen, and D. W. K. Ng, "Energy-efficient D2D overlaying communications with spectrum-power trading," IEEE Transactions on Wireless Communications, vol. 16, no. 7, pp. 4404-4419, 2017.

[27] F. Zhou, N. C. Beaulieu, Z. Li, J. Si, and P. Qi, "Energy-efficient optimal power allocation for fading cognitive radio channels: ergodic capacity, outage capacity, and minimum-rate capacity," IEEE Transactions on Wireless Communications, vol. 15, no. 4, pp. 2741-2755, 2016.

[28] S. Schaible, "Fractional programming," Zeitschrift für Operations Research, vol. 27, no. 1, pp. 39-54, 1983.

[29] I. Stancu-Minasian, Fractional Programming: Theory, Methods and Applications, Kluwer Academic Publishers, Dordrecht, The Netherlands, 1997.

[30] W. Dinkelbach, “On nonlinear fractional programming," Management Science, vol. 13, no. 7, pp. 492-498, 1967.

[31] S. P. Boyd and L. Vandenberghe, Convex Optimization, Cambridge University Press, Cambridge, UK, 2004.

[32] A. J. Goldsmith and P. P. Varaiya, "Capacity of fading channels with channel side information," IEEE Transactions on Information Theory, vol. 43, no. 6, pp. 1986-1992, 1997.

[33] Q. Wu, G. Y. Li, W. Chen, and D. W. K. Ng, "Energy-efficient small cell with spectrum-power trading," IEEE Journal on Selected Areas in Communications, vol. 34, no. 12, pp. 33943408, 2016.

[34] Q. Wu, W. Chen, M. Tao, J. Li, H. Tang, and J. Wu, "Resource allocation for joint transmitter and receiver energy efficiency maximization in downlink OFDMA systems," IEEE Transactions on Communications, vol. 63, no. 2, pp. 416-430, 2015. 


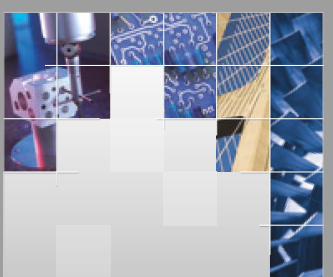

\section{Enfincering}
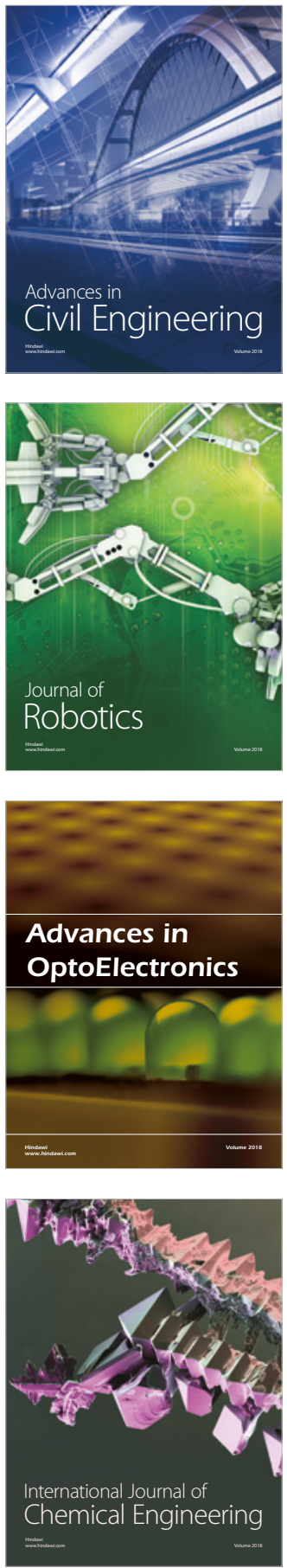

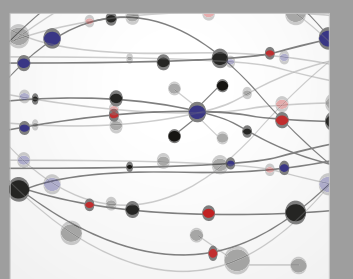

\section{Rotating \\ Machinery}

The Scientific World Journal

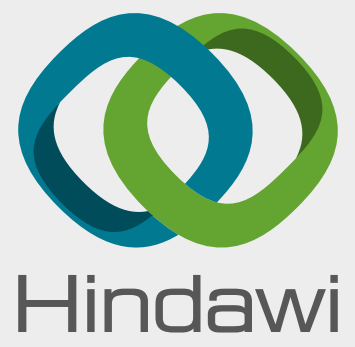

Submit your manuscripts at

www.hindawi.com
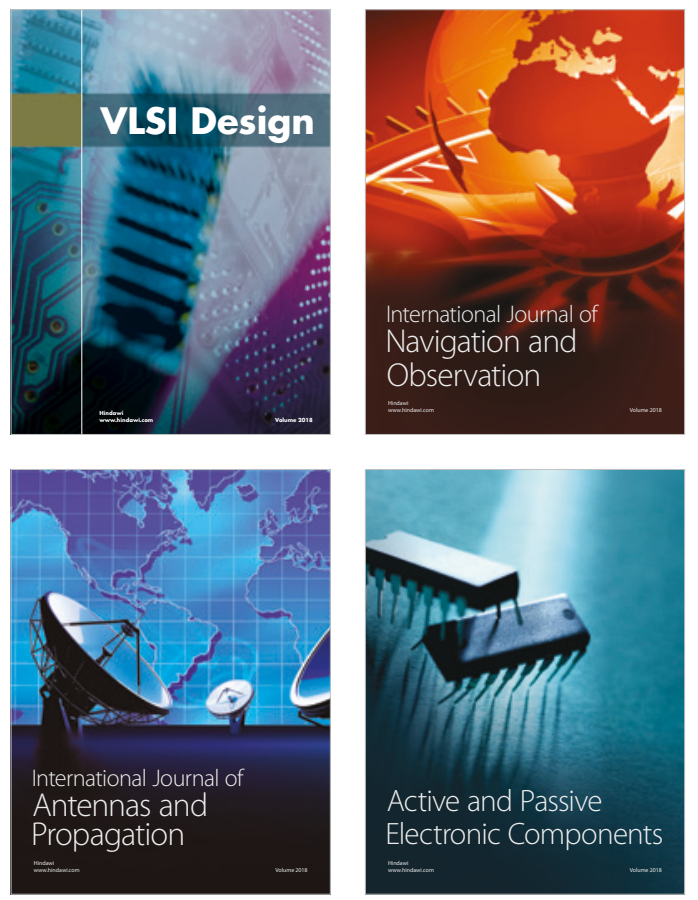
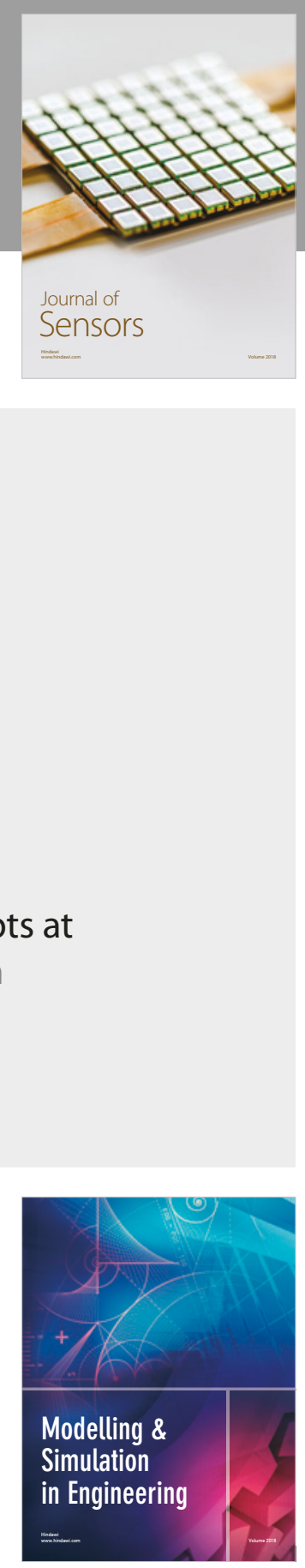

\section{Advances \\ Multimedia}
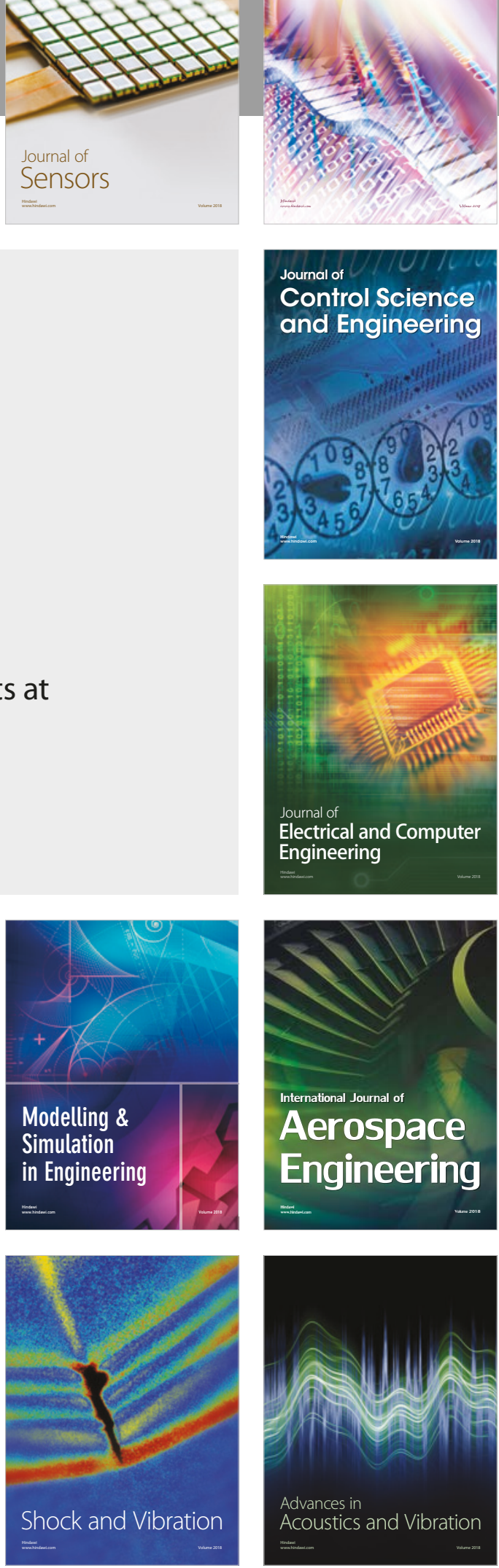